\title{
A Mechanistic Model of Bubble Entrainment in Turbulent Free Surface Flows
}

\author{
Alejandro M. Castro, Jiajia Li, Pablo M. Carrica* \\ IIHR-Hydroscience and Engineering \\ C. Maxwell Stanley Hydraulics Laboratory \\ The University of Iowa \\ Iowa City, IA 52242-1585 \\ ${ }^{*}$ Corresponding author \\ pablo-carrica@uiowa.edu \\ Ph: 1-319-335-6381 \\ FAX: 1-319-335-5238
}

\begin{abstract}
A framework for the development of models of bubble entrainment in free surface turbulent flows is presented. The framework uses mechanistic processes to model the stages involved in the entrainment of bubbles due to interaction of turbulence with a free surface. Entrainment is modeled as a chain of events for a bubble that is formed at the free surface, then pulled into the fluid against buoyancy, and interacts with vortices that break it up into smaller bubbles, or with other bubbles by coalescence. The main entrainment mechanism is modeled as a vortex/free surface interaction process that can entrain bubbles if the vortices located a given distance from the surface are strong enough. This approach overcomes limitations of approaches where the entrainment is determined only by turbulence parameters, which in the case of objects interacting with a free surface entrain bubbles on the boundary layers irrespective of the distance to the free surface. Depending on the computational fluid dynamics approach used to solve the flow, these processes may need different levels of modeling; more resolved approaches like large-eddy simulation with a volume of fluid method of the free surface will require less modeling complexity than a less resolved RANS method, since some of the involved processes of entrainment are directly accounted for. In this paper a standard RANS approach is used with the free surface modeled using a single-phase level set method, and models are presented for each of the relevant processes to produce a complete mechanistic model of turbulent bubble entrainment. The model was calibrated and tested for two relevant problems: a $2 \mathrm{D}+\mathrm{T}$ breaking wave in model scale, and the full scale bubbly flows around the US Navy Research Vessel Athena. For the second case a grid study is carried out to analyze grid convergence performance of the model. Comparisons with experimental data show that the model predicts well location and magnitude of entrainment.
\end{abstract}

Keywords: Bubble Entrainment, Polydisperse Bubbly Flow, Two-phase Flow, Free Surface, Wave Breaking, Ship Hydrodynamics. 


\section{Introduction}

Bubble entrainment in liquid flows is of importance for a variety of applications, both naturally occurring and as result of human action. The entrainment of bubbles brings oxygen to rivers and lakes, improving water quality and making healthy aquatic life possible. On the other hand, an excess of bubbles at depth can increase the concentration of nitrogen in water, causing excess of total dissolved gas and bubble fish disease (Politano et al., 2009). Aeration also plays an important role in the dynamics of oceanic oxygenation (Deane and Stokes, 1999; Callaghan et al., 2014) and in the mixing of rivers carrying domestic and industrial effluents with lake and coastal waters (Chen and Jirka, 1999; Baddour et al., 2006). Bubble entrainment is frequently an undesired side effect affecting the performance of hydraulic structures (Odgaard, 1986; Ervine, 1998), ship propellers (Walker and Johnson, 1991; Anthony and Willmarth, 1992) and precipitation reactors (Rousseaux et al., 2000; Assirelli et al., 2008). In other applications the controlled entrainment of bubbles may be beneficial, such as in already mentioned oxygenation situations or ship drag reduction (Kumagai et al., 2015).

Though air/water is the prevalent case, bubble entrainment can occur in other gas/liquid systems, being the cause of impurities and defects in the pouring of molten metal (Zhang and Thomas, 2003) and of performance issues in pool type liquid metal fast breeder reactors (LMFBR) where argon bubbles are entrained by the turbulent liquid sodium (Patwardhan et al., 2012). In this paper the air/water system is used as a generic for gas/liquid, though the entrainment model can in principle be used for any gas/liquid system.

Entrapment of air into the water causes bubble entrainment, and occurs through a variety of processes, including low pressure regions, such as vortex cores and trailing edges of appendages, collapse of air films caused by plunging jets. In these cases bubbles are entrained into 
the water when drag from the interface into the liquid overcomes buoyancy and surface tension forces that usually resist entrainment. Direct entrapment can also occur through collapse of cavities formed by plunging breaking waves, by drop impact, etc. The entrapped air is subject to the simultaneous action of breakup and coalescence, caused by turbulence, shear and buoyancy, resulting in a dynamic bubble size distribution. These various mechanisms obey completely different physics and should thus be modeled independently. For instance, in the case of a surface ship bow and shoulder waves often exhibit plunging jets and high levels of turbulence. The contact line between the hull and the free surface is subject to the highly turbulent ship boundary layer. The stern flow, mostly in transom sterns at lower Froude numbers, exhibits highly energetic largescale vortices and massive entrainment.

Though computers and numerical algorithms have made enormous progress in recent years, direct simulation of bubble entrainment in full scale applications is still many decades off. Figure 1 shows a diagram of the scales involved in bubble entrainment problems, where the largest scales are related to the size of the application and shown in the order of a hundred meters, representative for example of a nuclear reactor, a spillway or a ship. The smallest scales are related to the processes of bubble coalescence, involving film breakup processes and retarded Van der Waals forces where scales as small as $0.01 \mu \mathrm{m}$ are of importance. Bubbles and air cavities can have sizes between $20 \mu \mathrm{m}$ to $1 \mathrm{~cm}$. Consider the direct numerical simulation of a large scale problem such as the flow in a spillway or around a ship with a characteristic scale $L=100 \mathrm{~m}$. A uniform grid able to handle all these scales in a computational domain in the order of $3 L \times L \times L$ would need over $10^{30}$ grid points. If film breakup for coalescence is ignored, a minimum of 8 to 12 grid points are still needed to properly resolve the dynamics of bubbles' interfaces (Ling et al., 2015). Assuming optimistically that 5 grid points per bubble diameter are enough to resolve 
bubbles of approximately $25 \mu \mathrm{m}$ with a grid spacing of $5 \mu \mathrm{m}$, the total number of grid points required decreases to $2.4 \times 10^{22}$ points. Using 2.4 million points per core, still 10,000 trillion cores would be required for such computation, orders of magnitude more than all combined cores contained in all computers, phones, game consoles and tablets ever manufactured.

The time step size is also highly restrictive and has to be consistent with the time scales of the fastest processes, in the order of $\tau \approx\left(\rho R^{3} / \sigma\right)^{1 / 2}=10 \mu$ s or less for film breakup in bubble coalescence (Rice, 2006), though the CFL condition could be even more restrictive for such small grid sizes. Simulating the flow across 5 domain lengths at about $5 \mathrm{~m} / \mathrm{s}$ would then require some $10^{7}$ time steps. As a comparison, one of the largest computational fluid dynamics (CFD) simulations performed to date for air/water free surface problems have used about $2 \times 10^{10}$ grid points and a few thousands time steps (Dommermuth et al. 2014). The required improvement on machine capabilities, both in speed and memory, is in the order of $10^{23}$. If Moore's law of doubling computational power every two years holds, then it will take over 150 years to enable direct computation of bubbly flows on a large scale. Even if local grid refinement or localized hybrid approaches that resolve entrainment but model bubble/bubble interaction are used, direct simulation of entrainment in full scale applications is still decades away.

The direct numerical simulation of laboratory scale experiments however, has been achieved with today's supercomputing power (Wang et al., 2014; Dommermuth et al., 2014; Lubin and Glockner, 2015). For a simulation using $10^{9}$ grid points on a small scale tank of about $L=20 \mathrm{~cm}$ the grid size would be $200 \mu \mathrm{m}$ and the smallest bubble size that can be resolved would be $1 \mathrm{~mm}$ when considering only about 5 grid points per bubble diameter. However, for larger scale flows modeling of the bubble entrainment is needed. 
Most bubble transport models assume a given source and size distribution as a boundary or initial condition. In the modeling of Total Dissolved Gas (TDG), void fraction and mean size are estimated from previous experience or experiments (Politano et al., 2009). Similarly for bubbly columns void fraction and size distribution is imposed as an inlet boundary condition from available experiments or correlations (Chen et al., 2005).

In his pioneering work Baldy (1993) presents an air entrainment model for wind waves based on similarity arguments. The model assumes a linear relationship of the rate of air entrainment with turbulence dissipation $\varepsilon$, leading to a bubble size distribution proportional to $D^{-2}$ close to the free surface and to $D^{-4}$ away from it. A different dimensional analysis pursuing an inertial theory performed by Garrett et al. (2000) leads to a size distribution proportional to $\varepsilon^{-1 / 3} D^{-10 / 3}$ later confirmed by experiments by Deane and Stokes (2002), though no expression for the entrainment rate is proposed. Experimental studies of the air entrainment in the surf zone relate the amount of void fraction to the turbulent intensity (Cox and Shin, 2003; Mori et al., 2007). An entrainment model in the surf zone is proposed by Shi et al. (2010) as a volumetric source proportional to the production of turbulence by shear. Ma et al. (2011a) propose a model proportional to $\varepsilon$ as suggested by Baldy (1993), with the size distribution measured by Deane and Stokes (2002), and apply it to the simulation of entrainment in the surf zone using a polydisperse approach. More recently, Derakhti and Kirby (2014), applied Ma et al. (2011a) model to analyze the entrainment of bubbles by a breaking wave with a volume of fluid simulation that can resolve entrainment of the largest bubbles. All these models are appropriate for problems where solid surfaces are not present, like breaking waves, but fail if turbulence is generated by independent mechanisms away from the surface, as in the case of a ship's boundary layer. If turbulence is present away from the surface the model activates and unphysical production of bubbles occurs. 
Souders and Hirt (2004) propose to use local turbulent scales from an RNG turbulence model to trigger air entrainment, though little is known about the specifics of this implementation due to the proprietary nature of the work, though the authors report very encouraging results.

Extensive experimental studies for plunging jets are available and correlations based on the jet speed and fall length have been proposed (Ervine et al., 1980; Biń, 1993; Bonetto and Lahey, 1993). Even for this simple configuration the entrainment process is quite complex and is not yet fully understood, exhibiting large variability in results from different authors (Carrica et al. 2012). Using a simple fit with the jet velocity $U_{j}$ to the available experimental data, Ma et al. (2010) inject bubbles at the jet impact region in a two-phase CFD model.

Similar entrainment mechanisms occur during casting as surface oxide films fold into the bulk leading to cracks or porosity in the solidified product. Yue and Green (2012) propose an algorithm that identifies approaching interfaces and places particles as interfaces close onto each other. The particle properties were tuned to represent the behavior of double oxide films.

Bubble entrainment has attracted increasing attention to the ship hydrodynamics community. Bubbles naturally entrained by a ship advancing in a seaway change its wake's acoustic signature and affect the ship's detectability and vulnerability. Moraga et al. (2008) proposed a model that entrains bubbles at locations where the normal component of the velocity is larger than a specified threshold. The model is shown to perform well in steady cases. This model however, being a function of the liquid velocity, is frame dependent and predicts an unphysical entrainment of bubbles for traveling waves. Ma et al. (2011b) propose a model in which the entrainment rate is proportional to the product of the turbulent kinetic energy and gradient of the normal velocity in the direction of the free surface normal. Thought the gradient seems to be a good indicator of entrainment locations, the model constant is highly problem dependent and must 
be adjusted on a per-case basis (Carrica et al., 2012). A model based on energetic considerations is presented by Carrica et al. (2012) for turbulent free surface flows, the precursor for the modeling ideas presented in this work.

While the previously discussed entrainment models contributed significantly to advance the field, they suffer from a lack of mechanisms describing the entrainment processes in the derivation, being in most cases guided by phenomenological description. In addition, most models were not designed to depend only on local variables, in such a way that they can be implemented into CFD codes. In this paper we present a mechanistic models of turbulence-induced bubble entrainment. The goal is to develop a framework to generate entrainment models of various levels of sophistication depending on the complexity of the modelling of the basic physical processes used to describe the entrainment. At this stage of development simple (but mechanistic) models are used to close the system of equations arising from considering turbulence/free surface interaction.

\section{Modeling}

As discussed in the introduction, direct simulation of bubbly flows is beyond the capabilities of current computers for most applications of interest. One of the most advanced approaches to solve complex bubbly flows relies in polydisperse two-fluid models, where bubble populations are modeled in an averaged sense, introducing quantities like the concentration of bubbles and bubble size distributions. These modeling approaches, though expensive, are tractable with current supercomputing power, solving equations for the number density and velocity of each bubble size, resulting typically in one order of magnitude more variables than in single-phase computations. Other advanced methods include Eulerian-Lagrangian approaches, where bubbles 
are tracked in a Lagrangian manner. In general these approaches are less diffusive than approaches where bubbles are modeled in an Eulerian framework making them attractive for instance in problems where local concentration of bubbles needs to be resolved, but can become restrictively expensive if overly large number of bubbles are tracked, and two-way coupling can be challenging.

\subsection{Statistical Description of Polydisperse Flows}

This section briefly summarizes the two-fluid model used in this work to provide the mathematical framework for which the air entrainment model is developed. Further details can be found in Castro and Carrica (2013a; 2013b), Li et al. (2015), and references therein.

Though bubble transport is a complex problem and development is not yet mature, the twofluid approach has been studied extensively (Ishii, 1975; Drew and Passman 1999; Behzadi et al., 2004) and used successfully for a multitude of applications. In this work a two-fluid model in which water is the main continuous phase and bubbles are the disperse phase is used. The model is derived by ensemble averaging the momentum and continuity equations conditioned to be in either the continuous or disperse phase (Drew and Passman 1999). For the continuous phase this results in the two-fluid model momentum and continuity equations as

$$
\begin{gathered}
\frac{\partial \alpha_{c} \rho_{c} \boldsymbol{u}_{c}}{\partial t}+\nabla \cdot\left(\alpha_{c} \rho_{c} \boldsymbol{u}_{c} \otimes \mathbf{u}_{c}\right)=-\nabla \hat{p}_{c}+\nabla \cdot\left(2 \alpha_{c} \mu_{\mathrm{eff}, c} \nabla^{s} \mathbf{u}_{c}\right)+\alpha_{d} \rho_{c} g\left(1-\frac{\rho_{d}}{\rho_{c}}\right) \hat{k} \\
\frac{\partial \alpha_{c} \rho_{c}}{\partial t}+\nabla \cdot\left(\alpha_{c} \rho_{c} \boldsymbol{u}_{c}\right)=0
\end{gathered}
$$

where subscripts $c$ and $d$ are used to represent the continuous (water) and disperse (bubbles) phases, respectively. $\rho$ denotes a fluid density while $\mu_{e f f, c}$ denotes an effective dynamic viscosity 
obtained from a blended $k-\omega / k-\varepsilon$ model (Menter, 1994) extended to bubbly flow as described by Moraga et al. (2008). The amount of bubbles dispersed in the fluid is described by the volume fraction $\alpha_{d}$ while its complement $\alpha_{c}=1-\alpha_{d}$ is the liquid fraction. The use of the piezometric pressure $\hat{p}_{c}=p_{c}+\rho_{c} g \mathrm{z}$ in Eq. (1) is convenient in the description of free surface flows (Carrica et al. 2007). The last term in Eq. (1) models the effective buoyant forcing felt by the liquid as bubbles rise driven by gravity with acceleration $g$.

The free surface is computed as the zero level of a signed distance function $\phi$, positive in water, using a single-phase level set approach (Carrica et al. 2007),

$$
\frac{\partial \phi}{\partial t}+\boldsymbol{u}_{c} \cdot \nabla \phi=0
$$

In contrast to the standard level set, the single-phase level set is concerned with the solution of the flow field in water only. Advantages are that the interface remains sharp, computations are performed within a fluid with uniform properties and minor computations are required in air. Surface normals are simply computed as the gradient of the level set function $\hat{\boldsymbol{n}}=\nabla \phi$. Bubbles are present in the water, and computations in air are performed only to enforce appropriate boundary conditions at the free surface. Since Eq. (3) does not guarantee that $\phi$ remains a distance function throughout the computation, periodic reinitialization is needed (Carrica et al. 2007). Notice that upon reinitialization, $\phi$ is the distance to the free surface, a quantity needed by the bubble entrainment model.

The polydisperse bubbly phase is described by a number density function $f(m, \boldsymbol{r}, t)$, such that the number of bubbles per unit volume located at position $\boldsymbol{r}$ and at time $t$ with mass within $d m$ of $m$ is $f(m, r, t) \mathrm{d} m$. The bubble mass $m$ is a convenient choice to describe size since is not 
affected by pressure changes. Distributions in bubble diameter are obtained by the change of variables $D=(6 m / \pi \rho)^{1 / 3}$ using local values of gas density and assuming spherical bubbles, a good approximation for small bubbles for which surface tension dominates. The void fraction relates to the number density function by

$$
\alpha_{d}(\boldsymbol{r}, t)=\int_{0}^{\infty} d m \frac{m}{\rho_{d}} f(m, \boldsymbol{r}, t)
$$

The number density distribution obeys a Boltzmann-like transport equation (Carrica et al., 1999)

$$
\frac{\partial f}{\partial t}+\nabla \cdot\left(\boldsymbol{u}_{d} f\right)+\frac{\partial}{\partial m}(\dot{m} f)=\beta+\chi+S
$$

describing the transport of bubbles with a velocity $\boldsymbol{u}_{d}$ obtained from a momentum balance equation for each bubble size that includes the effects of drag, turbulent dispersion, virtual mass, lift and packing (for details see Castro and Carrica 2013b). Sources $\beta$ and $\chi$ in Eq. (5) account for the breakup and coalescence of bubbles respectively using the models of Lehr et al. (2002) for breakup and of Prince and Blanch (1990) for coalescence. The third term on the left of Eq. (5) accounts for bubble dissolution (Castro and Carrica 2013a). In this mathematical description air entrainment is modeled using the volumetric source $S$ in Eq. (5).

Assuming that inertia and shear stresses in the gas phase are negligible, the disperse phase momentum equation is simplified to

$$
-\alpha_{m} \nabla \hat{p}_{c}+p_{c} \nabla \alpha_{m}+\tilde{\boldsymbol{M}}_{m}(m)+\alpha_{m} \rho_{c} g\left(1-\frac{\rho_{d}}{\rho_{c}}\right) \hat{\boldsymbol{k}}=0
$$


where the void fraction distribution is defined as $\alpha_{m}(m, \boldsymbol{r}, t)=\frac{m}{\rho_{d}(m, \boldsymbol{r}, t)} f(m, \boldsymbol{r}, t)$. The interfacial force $\tilde{\boldsymbol{M}}_{m}$ is typically assumed to be a linear superposition of individual contributions

$$
\tilde{\boldsymbol{M}}_{m}=\boldsymbol{M}_{m}^{D}+\boldsymbol{M}_{m}^{V M}+\boldsymbol{M}_{m}^{L}+\boldsymbol{M}_{m}^{T D}
$$

representing, in order of appearance, the drag, virtual mass, lift and turbulent dispersion interfacial forces. Details on how these are modeled, as well as other details pertaining the free surface plolydisperse two-fluid model summarized by Eqs. (1), (2), (5) and (6) can be found in Castro and Carrica (2013a, 2013b).

\subsection{Bubble Entrainment Model}

Figure 2 shows some physical processes that induce bubble entrainment. A bubble is entrained into the flow by direct entrapment, by vortices interacting with the surface, or other processes. Two examples of direct entrapment are a drop that impacts the surface trapping bubbles that cannot escape the connection of the drop with the underlying fluid, and a breaking wave that as it closes at plunging traps the air contained in the so called pipeline. Vortices interacting with a free surface cause surface deformations that may result in bubble entrainment. The entrainment onset and intensity is determined by a competition between forces dragging bubbles down and opposing surface tension and buoyancy forces. Vortices tangential and normal (funnels) to the surface behave differently, with normal vortices entraining bubbles deeper. Though vortices interacting with a free surface trigger other processes (spilling waves, entrapment, etc.), vortex/free surface interaction can be considered a macroscopic phenomenon that is an entrainment process in itself. 


\subsubsection{A General Framework}

Entrainment is modeled as a chain of events for a bubble that is formed at the free surface, then pulled into the fluid against buoyancy, and interacts with vortices that break it up into smaller bubbles, or with other bubbles by coalescence. The model estimates the probability of occurrence for each of these events and combines them into a single air entrainment volumetric source. In this way, the proposed framework describes the very complex entrainment process as a series of simpler stages, which could be possibly modeled independently using simple models with closed mathematical descriptions.

This framework is developed under the following set of idealizations and assumptions:

1) The total rate of air entrainment can be obtained as the linear superposition of contributions of individual vortices underneath the free surface in the turbulent field.

2) The turbulence underneath the free surface can be described as a collection of individual vortices of characteristic size $\ell$ at depth $z^{\prime}$, denoted by $V=\left\{\ell, z^{\prime}\right\}$.

3) These vortices are pictured as separate entities with energy content $e_{\ell}$ and with a population in number given by a number density $n_{\ell}$ for vortices of size $\ell$.

4) The particular geometrical shape of this vortices (tube, whirlpool, loop) is not as important as their energy content $e_{\ell}$, determined from their characteristic spin velocity $u_{\ell}$ to be determined from the energy spectrum.

The model presented in this work is mostly motivated by premise 1), a very strong modeling assumption inspired by previous work on bubble breakup by Luo and Svendsen (1996) and later followed by others (Lehr et al., 2002; Wang et al., 2003), which has led to successful breakup models in use today. 
Consider an infinite free surface on the $x-y$ plane underneath which a turbulent field with zero mean velocity drives the entrainment of bubbles. The turbulent field is assumed to be only a function of $z$ normal to the free surface, which on average is located at $z=0$. A single vortex in this turbulent field of characteristic size $\ell$ at depth $z^{\prime}$ is depicted in Fig. 3. Its rotational motion induces a deformation on the free surface and bubble entrainment occurs if the surface deforms beyond a critical value. The rate of bubble entrainment by this single vortex in cubic meters of air per second is denoted as $\dot{Q}_{\ell}(\mathrm{V})$.

Vortex sizes span a wide range of scales from the Kolmogorov scale $\eta$ to several integral length scales $L_{11}$. The spectral density of vortices $n_{\ell}(\ell)$ is defined such that $n_{\ell}(\ell) d \ell d V^{\prime}$ is the number of vortices within a differential volume $d V^{\prime}$ with characteristic length within $d \ell$ of $\ell$. Consider now a test slice in Fig. $3 d z^{\prime}$ thick with area $A$ such that $\ell<<\sqrt{A}$ on a plane parallel to the mean free surface at depth $z^{\prime}$. From assumption 1) the total amount of air entrained by vortices with size within $d \ell$ of $\ell$ in the volume $d V^{\prime}=A d z^{\prime}$ is $\dot{Q}_{\ell}(\mathrm{V}) n_{\ell}(\ell) d V^{\prime} d \ell$. The number of entrained bubbles per unit time can be obtained by dividing this result by the mean bubble volume at the free surface $\bar{v}_{0}$.

Bubbles formed at the free surface by a vortex $\mathrm{V}$ have a formation size distribution $p_{0}(D \mid \mathrm{V})$. The amount of bubbles formed at the free surface with diameter in $\left(D_{0} ; D_{0}+d D_{0}\right)$ by vortices within $d z^{\prime}$ of depth $z^{\prime}$ with a size in $(\ell ; \ell+d \ell)$ is then

$$
d \dot{n}\left(D_{0} \mid \mathrm{V}\right)=\frac{1}{\bar{v}_{0}} \dot{Q}_{\ell}(\mathrm{V}) n_{\ell}(\ell) d V^{\prime} d \ell p_{0}\left(D_{0} \mid \mathrm{V}\right) d D_{0}
$$

Equation (8) describes the rate of bubbles formed at the free surface before they are dragged down to depth into the fluid, being effectively entrained. Since their inception at the free surface, 
bubbles that travel to a depth $z$ may breakup by interacting with turbulent eddies or coalesce by interacting with other bubbles as depicted in Fig. 4. The probability of finding a bubble of size $D$ at depth $z$ after generation at the free surface with size $D_{0}$ is denoted as the history size distribution $p_{h}\left(D \mid D_{0}, z\right)$.

Figure 4 suggests a way to estimate $p_{h}$ by considering a 0D model of breakup and coalescence with an initial monodisperse bubble size distribution $D_{0}$ left to evolve for a time of travel $t_{z}$. This time of travel can be estimated from predicted turbulent quantities by either considering random walk or simple transport depending on the integral length scale of turbulence $L_{11}$.

Lastly, bubbles are effectively filtered by the pull of gravity inducing a terminal velocity which is larger for larger bubbles. This effect is taken into account by introducing $p_{z}(z \mid D) d z$, the probability of a bubble with size $D$ being pushed down to within $d z$ of a depth $z$, not necessarily equal to the vortex depth $z^{\prime}$.

Altogether, the rate of bubbles with sizes in $(D ; D+d D)$ entrained to within $d z$ of $z$ by vortices with sizes in $(\ell ; \ell+d \ell)$ located within $d z^{\prime}$ of depth $z^{\prime}$ is

$$
d \dot{n}_{e}=\frac{\dot{Q}_{\ell}(\mathrm{V})}{\bar{v}_{0}} n_{\ell}(\ell) d \ell d V^{\prime} p_{0}\left(D_{0} \mid \mathrm{V}\right) d D_{0} p_{h}\left(D \mid D_{0}, z\right) d D p_{z}(z \mid D) d z
$$

According to Eq. (9) bubbles are not directly formed with size $D$ at depth $z$, but formed at the free surface with size $D_{0}$ and transported to depth $z$ while breakup, coalesce and buoyancy effectively filters them to a final size $D$. 
The total source of bubble entrainment is obtained by integration of Eq. (9) within appropriate limits. Assuming that vortices of size $\ell$ can only entrain bubbles with diameter $D_{0}<\ell$ , integration on $D_{0}$ is performed in $(0, \ell)$. To avoid singularities later in the modeling of single vortices, it is assumed that vortices are fully immersed in the liquid, or $\ell<2 z^{\prime}$, and thus $\ell$ is integrated over $\left(0,2 z^{\prime}\right)$. Finally, the entire turbulent field underneath the free surface is considered by integrating in $z^{\prime}$. The probability of finding a bubble at a given depth is related to the competitive process between buoyancy and turbulent mixing which is not linked to a particular vortex size but to the turbulent field as a whole. Therefore it is assumed that $p_{z}(z \mid D)$ does not depend on the vortex variables and can be factored out. Consequently, the total amount of bubble entrainment is evaluated as

$$
d S(D, z)=\underbrace{d z A d D}_{d V} \frac{1}{\bar{v}_{0}} p_{z}(z \mid D) \int_{0}^{\infty} d z^{\prime} \int_{0}^{2 z^{\prime}} d \ell \dot{Q}_{\ell}(\mathrm{V}) n_{\ell}(\ell) \int_{0}^{\ell} d D_{0} p_{0}\left(D_{0} \mid \mathrm{V}\right) p_{h}\left(D \mid D_{0}, z\right)
$$

The volumetric entrainment source as needed by the model in Eq. (5) is obtained by noticing that $d V=A d z$ constitutes the differential test volume where bubbles are entrained. Therefore

$$
S(D, z)=\frac{d S(D, z)}{d V d D}=\frac{1}{\bar{v}_{0}} p_{z}(z \mid D) \int_{0}^{\infty} d z^{2} \int_{0}^{2 z^{\prime}} d \ell \dot{Q}_{\ell}(\mathrm{V}) n_{\ell}(\ell) \int_{0}^{\ell} d D_{0} p_{0}\left(D_{0} \mid \mathrm{V}\right) p_{h}\left(D \mid D_{0}, z\right)
$$

Notice that Eq. (11) provides a modeling framework rather than a closed model for air entrainment by allowing the modeler to specify different approximations for each of the individual terms describing a given process. The remainder of this section is devoted to the development of simple approximations for each of the factors in Eq. (11) to arrive to a closed form of an entrainment model for a RANS CFD solver in which the free surface is modeled with a single- 
phase approach (Carrica et al., 2007). However, each of these approximations could be further improved based either on new theories, experimental data, or highly resolved simulations of flows with bubble entrainment. In addition, other CFD solution approaches may better resolve some of the terms in Eq. (11) and thus require simpler or no modeling at all.

\subsubsection{Turbulent Scales}

Pope (2000) proposes an analytical turbulence spectrum for homogeneous turbulence, fully specified in terms of the turbulent kinetic energy $k_{t}$ and turbulent dissipation $\varepsilon_{t}$

$$
\begin{aligned}
& E(\kappa)=C \varepsilon_{t}^{2 / 3} \kappa^{-5 / 3} f_{L}^{5 / 3+p_{0}}(\kappa L) f_{\eta}(\kappa \eta) \\
& f_{L}(\kappa L)=\frac{\kappa L}{\left[(\kappa L)^{2}+c_{L}\right]^{1 / 2}} \\
& f_{\eta}(\kappa \eta)=e^{-\beta\left\{\left[(\kappa \eta)^{4}+c_{\eta}^{4}\right]^{1 / 4}-c_{\eta}\right\}}
\end{aligned}
$$

where $\kappa=2 \pi / \ell$ is the wavenumber, $L=k_{t}^{3 / 2} / \varepsilon_{t}$ and $\eta=\left(v^{3} / \varepsilon_{t}\right)^{1 / 4}$ is the Kolmogorov scale with model parameters $C=1.5, p_{0}=2, c_{L}=6.78, \beta=5.2$ and $c_{\eta}=0.4$. Turbulent kinetic energy, dissipation and integral length scale are obtained from the spectrum as

$$
\begin{aligned}
& k_{t}=\int_{0}^{\infty} E(\kappa) d \kappa \\
& \varepsilon_{t}=\int_{0}^{\infty} 2 \nu \kappa^{2} E(\kappa) d \kappa \\
& L_{11}=\frac{3 \pi}{4 k_{t}} \int_{0}^{\infty} \frac{E(\kappa)}{\kappa} d \kappa
\end{aligned}
$$

with the integral length scale $L_{11} \approx 0.43 L$ for turbulent flows with $R e_{\lambda}>150$ where $R e_{\lambda}$ is the Taylor microscale based Reynolds number. To characterize the limits of the inertial subrange, the 
transition length from the energy containing range to the inertial range $L_{E I}$ is defined where $f_{L}^{5 / 3+p_{0}}\left(L_{E I}\right)=0.95$, and the transition length from the inertial range to the dissipation range is defined such that $f_{\eta}\left(L_{I D}\right)=0.95$. This results in the limits $L_{E I} \approx 0.945 L_{11} \approx L_{11}$ and $L_{I D} \approx 28 \eta$.

In the inertial subrange the RMS velocity of eddies with size $\ell$ follows the law $u_{\ell} \approx 1.41\left(\varepsilon_{t} \ell\right)^{1 / 3}$ (Kuboi et al., 1972). To extend this relation into the energy containing range, it is assumed that $u_{\ell} \propto \kappa$ and that a smooth transition with a functional form similar to the one for $f_{L}$ in Eq. (12) is valid

$$
\begin{aligned}
u_{\ell} & =\sqrt{2}(2 \pi)^{1 / 3}\left(\frac{\varepsilon_{t}}{\kappa}\right)^{1 / 3} f_{L}^{1 / 3+1} \\
& =\sqrt{3}(2 \pi)^{1 / 3} \frac{u_{0}}{(\kappa L)^{1 / 3}} f_{L}^{4 / 3}
\end{aligned}
$$

where for homogeneous and isotropic turbulence the RMS of the velocity fluctuations is $u_{0}=\left(2 k_{t} / 3\right)^{1 / 2}$.

As an example, CFD estimations at the highly turbulent stern of Athena R/V, a $47 \mathrm{~m}$ Asheville-class patrol, advancing at 10.5 knots are summarized in Table 1 and shown in Fig. 5 along with the turbulent spectrum from Eq. (12) and the RMS velocity from Eq. (14). Different vertical axes are used to show $E(\kappa)$ and $u_{\ell}$ in the same plot. In Table 1, Hinze's diameter is defined as (Hinze, 1955)

$$
D_{H}=0.725\left(\frac{\sigma}{\rho_{c}}\right)^{3 / 5} \frac{1}{\varepsilon_{t}^{2 / 5}}
$$


Bubbles with a diameter smaller than $D_{H}$ are strongly stabilized by surface tension and very rarely break. However, bubbles smaller than $D_{H}$ can be formed by the breakup of larger bubbles and by highly energetic impact processes.

Higher order models including second-order turbulent structures and shear are available (Mann, 1994). Mann's model however, still uses a homogeneous and isotropic model spectrum to specify the energy content. It is important to note however, that the key factor in the development of the present model is the energy content of turbulent structures rather than their particular shape or geometric configuration. It is for this reason that anisotropy is not considered at this stage. Turbulence anisotropy has the potential to induce coherent structures such as funnels which are observed to occur specially at low ship speeds. Future refinements to the model will attempt to include these structures and establish their importance.

\subsubsection{Vortex Density}

The density of vortices $n_{\ell}$ in Eq. (11) is modeled following the ideas of Luo and Svendsen

(1996) by considering vortices as spheres of diameter $\ell$ with mean kinetic energy $e_{\ell}=\frac{1}{2} \rho \frac{\pi}{6} \ell^{3} u_{\ell}^{2}$ . If $n_{\ell}(\ell) d \ell$ is the number of vortices per unit volume with sizes in $(\ell ; \ell+d \ell)$ then

$$
\Lambda\left(\alpha_{d}\right) E(\kappa) d \kappa=n_{\ell} e_{\ell} d \ell
$$

where the fraction of available liquid is considered in the factor $\Lambda\left(\alpha_{d}\right)=1-\alpha_{d}$. From Eq. (16) the vortex density is determined with the use of the spectrum in Eq. (12)

$$
n_{\ell}(\ell)=0.8413 \frac{1}{\ell^{4}} f_{L} \Lambda\left(\alpha_{d}\right)
$$


The vortex density is proportional to $\ell^{-4}$ for $\ell<<L$ and to $\ell^{-5}$ for $\ell>>L$.

\subsubsection{Single Vortex Bubble Entrainment}

In this section a model is proposed for the entrainment caused by vortices interacting with the free surface, $\dot{Q}_{\ell}(\mathrm{V})$ in Eq. (11). Proper modeling of the entrainment caused by individual vortices interacting with the free surface is important. For the case of entrainment caused by objects interacting with a free surface, like surface-piercing cylinders or ships, turbulence is generated by boundary layers and breaking waves. Models designed to predict entrainment on breaking waves, as those by Baldy (1993) and Derakhti and Kirby (2014), use the turbulent kinetic energy dissipation as a metric to determine entrainment, and would thus predict entrainment in boundary layers irrespectively of the distance to the free surface. In addition, the highly dissipative small vortices in a boundary layer would entrain air regardless of their size. A complete model should include the Hinze diameter to limit entrainment for very small vortices and the turbulent kinetic energy to identify vortices too large to induce surface deformations leading to entrainment.

To prevent that physical behavior, the entrainment has to be based on mechanisms of interaction between vortices and free surface, necessarily involving the ability of the vortices to produce entrainment as the distance to the free surface changes.

A simple analytical model based on potential flow theory is formulated. In this model an inviscid vortex line of diameter $\ell$ is placed underneath a flat free surface at a depth $z^{\prime}$ and its image at $-z^{\prime}$ to satisfy an approximate free surface boundary condition at $z=0$ as depicted in Fig. 6. The strength of the isolated vortex is such that at $\ell / 2$ away from the vortex the velocity is $u_{\ell}$. This leads to the stream function 


$$
\psi=\frac{u_{\ell} \ell}{2}\left[\ln \left(\sqrt{x^{2}+\left(z^{\prime}+z\right)^{2}}\right)-\ln \left(\sqrt{x^{2}+\left(z^{\prime}-z\right)^{2}}\right)\right]
$$

from where velocity can be computed and used with Bernoulli's equation to obtain the pressure

$$
p(x, z)=p_{a}+\rho g z-\frac{1}{2} \rho u^{2}(x, z)
$$

with $p_{a}$ the atmospheric pressure. Using the dynamic pressure induced at $z=0$ the free surface elevation $h(x)$ is then approximated by finding the level of $p(x, h(x))=p_{a}$

$$
h(x)=\frac{u^{2}(x, 0)}{2 g}=\frac{\left(u_{\ell} \ell\right)^{2}}{2 g} \frac{z^{\prime 2}}{\left(z^{\prime 2}+x^{2}\right)^{2}}
$$

Based on observations made by Duncan (2001) for breaking waves, the model assumes that air entrainment occurs when the slope of the free surface deformation reaches a critical angle of $18^{\circ}$ or a critical slope $s_{\mathrm{cr}}=0.325$. The slope $s(x)=\partial h(x) / \partial x$ is obtained from Eq. (20) as

$$
\tilde{s}=\frac{1}{2 F r_{\ell}^{2}}\left(\frac{z^{\prime}}{\ell}\right)^{3} s=\frac{\tilde{x}}{\left(1+\tilde{x}^{2}\right)^{3}}
$$

with $\tilde{x}=x / z^{\prime}$ and the vortex Froude number defined as $F r_{\ell}=u_{\ell} / \sqrt{g \ell}$.

Essentially, and according to this model, vortices underneath the free surface will form lines of entrainment at the free surface where the critical slope for entrainment is reached. This simplified representation enables computation of the entrainment rate across each of these onedimensional entities and total rate of entrainment by superposition. A model for the geometrical configuration of these lines is then needed. Levy et al. (2011) studied the formation of vortex rings or flukeprints generated by whales as they swim underwater and the potential to entrain air at their edges. This observation inspired the visualization of vortices of size $\ell$ inducing entrainment rings 
of diameter $D_{s}$ at the free surface deforming along their circumference according to the approximation in Eq. (21).

In analogy to the analysis carried by Carrica et al. (2012) for plunging jets, the entrainment across this ring is modeled as proportional to the surface roughness $\delta$ and inwards surface velocity $u_{s}$ as

$$
\dot{Q}_{\ell}(\mathrm{V})=S_{\ell 0} D_{s} \delta u_{s}
$$

with $S_{\ell 0}$ hopefully a universal enough constant for a wide range of bubble entrainment problems. The surface velocity at the ring is estimated from the potential solution as $u_{s}(\ell, z)=u_{\ell} \ell / z$. According to Hunt (1984), the free surface roughness can be modeled from a balance between the potential energy of the surface deflection and the kinetic energy of the vortex leading to $\delta \propto u_{s}^{2} / g$

For vortices too close to the free surface Eq. (21) predicts unphysically large deformations, and the free surface will starting breaking limiting the physically possible maximum slope. To prevent this, the maximum slope is arbitrarily limited to $45^{\circ}$, or $s_{M} \leq 1.0$. The depth at which a vortex induces this maximum slope is denoted by $z_{m}$, computed from Eq. (21) with $s=s_{M}$

$$
z_{m}=0.803 \ell \mathrm{Fr}^{2 / 3}
$$

Note that the choice of the limiting value for $s_{M}$ should be supported by experimental data. Unfortunately, there is no bubble entrainment data for the interaction of vortices with a free surface, nor for any other simple relevant problem. To some extent, the value of the limit $s_{M} \leq 1.0$ can be considered a calibration constant. However, if the assumption of a maximum angle represents a good approximation of reality regardless of the actual numerical value of this angle, 
the scaling presented in Eq. (23) will convey the important physics. Equation (23) is an elegant scaling law that could be directly compared with experiments if available.

The maximum vortex ring size $D_{s M}$ is determined from Eq. (21) with $x=D_{s M} / 2$ and $Z=Z_{m}$ as

$$
D_{s M}=1.914 \ell \mathrm{Fr}_{\ell}^{2 / 3}
$$

When the vortex is not strong enough to induce the minimum slope $s_{\mathrm{cr}}$, Eq. (21) does not have a solution and therefore $D_{s}=0$ is used for convenience. The onset depth at which the maximum slope at the free surface reaches $S_{\text {cr }}$ is

$$
z_{e}=1.168 \ell \mathrm{Fr}^{2 / 3}
$$

Vortices farther than $z_{e}$ from the free surface are not able to induce deformation slopes larger than $S_{\text {cr }}$ and therefore do not entrain bubbles. Equations (23)-(25) suggest the length scale $\ell \mathrm{Fr}^{2 / 3}$ as a good reference scale to collapse single vortex results. Experimental data to validate Eq. (25) would be very beneficial in understanding the entrainment processes of a single vortex interacting with a free surface.

Finally, if the onset depth is used as a reference length the entrainment diameter can be written as

$$
\frac{D_{s}}{\mathrm{z}_{e}}=\tilde{D}_{s}\left(\mathrm{z} / \mathrm{z}_{e}\right)=\left\{\begin{array}{cc}
0 & \mathrm{Z} / \mathrm{z}_{e}>1 \\
2 \frac{\mathrm{z}}{\mathrm{Z}_{e}} \tilde{x}\left(0.824 s_{\mathrm{cr}}\left(\frac{\mathrm{z}}{\mathrm{z}_{e}}\right)^{3}\right) & 0.688<\mathrm{z} / \mathrm{z}_{e}<1 \\
1.615 & \mathrm{z} / \mathrm{z}_{e}<0.688
\end{array}\right.
$$


This profile is shown in Fig. 7 together with the onset of entrainment depth shown as a function of $F r_{\ell}$ as well as a function of vortex size $\ell$ for the parameters in Table 1

These results are used in Eq. (22) to obtain the single vortex entrainment rate model

$$
\dot{Q}_{\ell}(\mathrm{V})=S_{\ell 0} \frac{u_{\ell}^{3} \ell^{3}}{g z_{e}^{2}} F\left(z / z_{e}\right), \quad F\left(z / z_{e}\right)=\left(\frac{z_{e}}{z}\right)^{3} \tilde{D}_{s}\left(z / z_{e}\right)
$$

\subsubsection{Probability of Breakage and Formation Size Distribution}

In this section the formation size distribution $p_{0}(D \mid \mathrm{V})$ in Eq. (11) is discussed. The probability of breakage $P_{B}(D \mid \mathrm{V})$ is defined as the probability of finding a vortex of size $\ell$ with the energy to form a bubble of size $D$ by breakage of the surface. The probability of breakage was introduced by Luo and Svendsen (1996) and later used by Lehr et al. (2002) in the context of bubble breakup by turbulence. In the work of Lehr et al. (2002) a bubble breaks up if the dynamic pressure from the fluid overcomes the stabilizing surface tension force

$$
\frac{1}{2} \rho_{c} u^{2} \geq \frac{2 \sigma}{D}
$$

or equivalently, a bubble of size $D$ can be formed by a vortex of size $\ell$ if $u>u_{\min }=\sqrt{4 \sigma / \rho_{c} D}$.

Lehr et al. (2002) proposed a model for the probability of breakage $P_{B}$ which is an exponential function of $u_{\min } / u_{\ell}$. In this work a mechanistic derivation of $P_{B}$ based on finding the probability of the velocity fluctuations having the energy to overcome surface tension is presented. Fluctuations in velocity for vortices with a characteristic size $\ell$ approximately follow a Gaussian distribution with zero mean and a standard deviation equal to the characteristic velocity $u_{\ell}$, given 
by Eq. (14). If each component of the velocity vector follows a Gaussian distribution then its magnitude $u$ follows a Maxwell distribution

$$
p(u)=\sqrt{\frac{2}{\pi}} \frac{u^{2}}{u_{\ell}^{3}} e^{-\frac{1}{2}\left(\frac{u}{u_{\ell}}\right)^{2}}
$$

Using Eq. (28) the probability of breakage can be estimated from the probability distribution in Eq. (29)

$$
\begin{aligned}
P_{B}(D \mid \mathrm{V}) & =P\left(u>u_{\min }\right)= \\
& =\int_{u_{\min }}^{\infty} d u p(u)=\operatorname{erfc}(x)+\frac{2}{\sqrt{\pi}} x e^{-x^{2}}
\end{aligned}
$$

where

$$
\begin{aligned}
& x=\frac{u_{\min }}{u_{\ell} \sqrt{2}}=\sqrt{\frac{2}{W e\left(D, u_{\ell}\right)}} \\
& W e\left(D, u_{\ell}\right)=\frac{\rho_{c} D u_{\ell}^{2}}{\sigma}
\end{aligned}
$$

This model predicts a probability between $50 \%$ to $90 \%$ for Weber numbers from 1.7 to 6.8 . Critical Weber numbers in the range 3-4.7 have been measured by Lewis and Davidson (1982) and by Martínez-Bazán et al. (1999).

Consider the probability of forming bubbles between $D$ and $D+d D$ by a vortex of size $\ell$. This is, by definition, given by the formation size distribution as $p_{0}(D \mid \mathrm{V}) d D$. To form such bubble sizes the vortex velocity needs to be higher than $u_{\text {min }}(D+d D)$ but still smaller than $u_{\text {min }}(D)$ . This can be written in terms of the probability of breakage as 


$$
\begin{aligned}
& p_{0}(D \mid \mathrm{V}) d D=P\left(u_{\text {min }}(D+d D)<u<u_{\text {min }}(D)\right)= \\
& =P_{B}(D+d D \mid \mathrm{V})-P_{B}(D \mid \mathrm{V}) \approx \frac{d P_{B}(D \mid \mathrm{V})}{d D} d D
\end{aligned}
$$

and consequently the formation size distribution introduced in Eq. (8) is normalized by the probability of breakage as

$$
\int_{0}^{\ell} d D_{0} p_{0}\left(D_{0} \mid \mathrm{V}\right)=P_{B}(\ell \mid \ell)
$$

Notice that the introduction of surface tension effects in the model was delayed until now, allowing for a simple single vortex model without surface tension.

\subsubsection{Probability of Finding a Bubble at a Given Depth}

In this section a model for the probability $P_{z}(z \mid D)$ of a bubble with size $D$ being transported down to within $d z$ of a depth $z$ is proposed, Consider an idealized isotropic turbulent flow with zero mean velocity and mean free surface located at $z=0$. An equilibrium is eventually reached in which the entrainment rate is balanced by bubbles lost as they rise and escape through the free surface. To find the equilibrium profile the steady state transport equation for $f(D)$ is solved from Eq. (5), neglecting breakup and coalescence

$$
-V_{T}(D) f(D, t)=v_{t}(z) \frac{\partial f(D, t)}{\partial z}
$$

where the turbulent viscosity $v_{t}$ is a function of depth $z$ only since the turbulent field is assumed homogeneous in the horizontal direction, and the terminal velocity $V_{T}$ is a function of the bubble size. The solution to this equation can be obtained analytically for several turbulent viscosity 
profiles. A model for homogeneous turbulence with constant $v_{t}$ leads to the unphysical result of non-zero bubble densities far from the free surface, especially for small bubbles. In reality turbulence near a free surface decays very rapidly for depths larger than a characteristic size in the order of the integral length scale. Therefore, a more realistic model for $v_{t}$ is adopted

$$
v_{t}(z)=v_{t 0}\left(1-\frac{z}{L_{11}}\right), \quad z<L_{11}
$$

where the turbulent viscosity decays from $v_{t 0}$ at the free surface to zero at $L_{11}$. The probability $P_{z}(z \mid D)$ is modeled by normalizing the size distribution $f(D)$ as

$$
P_{z}(z \mid D)=\frac{f(z, D)}{\int_{0}^{\infty} d z f(z, D)}
$$

which for the linearly decreasing turbulent profile results in

$$
P_{z}(z \mid D)=\frac{1}{L_{z}}\left(1-z / L_{11}\right)^{L_{11} / L_{D}}
$$

with $L_{D}(D)=v_{t 0} / V_{T}(D)$ and $L_{z}=\left(L_{11}^{-1}+L_{D}^{-1}\right)^{-1}$.

\subsection{Further Simplifications}

Even though the models developed for each factor in Eq. (11) are quite simple, it is difficult to arrive to a closed analytical expression given the complex functional forms of the integrands. This section presents a series of approximations used to arrive to a closed analytical form that can be implemented in CFD codes. 


\subsubsection{History Size Distribution}

The history size distribution $p_{h}\left(D \mid D_{0}, z\right)$ in Eq. (11) is discussed next. A model for the history size distribution would require to integrate the Boltzmann Eq. (5) to include breakup and coalescence effects as entrained bubbles travel from the surface into the liquid. This integration is a non-trivial task even for a known turbulent field. To simplify the model and as a first approximation, it is assumed that breakup and coalescence reach equilibrium instantly. This assumption implies that no matter what the initial bubble size $D_{0}$ is, the history size distribution $p_{h}$ approaches an equilibrium size distribution that depends on bubble size only, i.e. $p_{h}\left(D \mid D_{0}, z\right) \approx p_{h}(D)$. This conveniently allows to factor out $p_{h}$ from Eq. (11) as

$$
S(D, z)=\frac{1}{\bar{v}_{0}} p_{z} p_{h} \int_{0}^{\infty} d z^{\prime} \int_{0}^{2 z^{\prime}} d \ell \dot{Q}_{\ell} n_{\ell} \int_{0}^{\ell} d D_{0} p_{0}
$$

where for convenience arguments are omitted to simplify notation. Further, the innermost integral can be evaluated to $P_{B}(\ell \mid \ell)$ according to Eq. (33). Finally, it is convenient to change the order of integration between $\ell$ and $z^{\prime}$ leading to

$$
S(D, z)=\frac{1}{\bar{v}_{0}} p_{z} p_{h} \int_{0}^{\infty} d \ell n_{\ell} P_{B} \int_{\ell / 2}^{\infty} d z^{\prime} \dot{Q}_{\ell}
$$

where the computation of the innermost integral is treated in the next section.

\subsubsection{Single Vortex Air Entrainment}

Using the change of variables $u=z / z_{e}$ the integral of $\dot{Q}_{\ell}$ in $z^{\prime}$ in Eq. (39) can be written 


$$
\begin{aligned}
& \int_{\ell / 2}^{\infty} d z^{\prime} \dot{Q}_{\ell}(\ell, z)=S_{\ell 0} \frac{u_{\ell}^{3} \ell^{3}}{g z_{e}} I_{Q}\left(F r_{\ell}\right) \\
& I_{Q}\left(F r_{\ell}\right)=\int_{u_{\min }}^{1} d u F(u) \quad, \quad u_{\min }=\frac{0.428}{F r_{\ell}^{2 / 3}}
\end{aligned}
$$

with $F(u)$ as defined in Eq. (27). The integral $I_{Q}$ can be performed analytically to within a 5\% error approximating the ring diameter from Eq. (26) by $D_{s} / z_{e} \approx 1.615 H\left(1-z / z_{e}\right)$ with $H(x)$ the Heaviside function. The result is

$$
I_{Q}\left(F r_{\ell}\right) \approx 0.808\left(5.46 F_{\ell}^{4 / 3}-1\right)
$$

Figure 8 shows that the integral $I_{Q}$ as a function of the vortex Froude number goes to zero for $F r_{\ell}<0.28$. Since $u_{\ell} \propto \ell^{1 / 3}$, then the Froude number scales as $F r_{\ell} \propto \ell^{-1 / 6}$; this result implies that large vortices do not entrain air, because they generate long wavelength waves that are not steep enough,. $I_{Q}$ increases for smaller vortices (larger Froude numbers). However, the total integral in Eq. (40) goes to zero as it is multiplied by the factor $u_{\ell}^{3} \ell^{3} / z_{e}$. This result is expected, since for smaller vortices $u_{s}, D_{s}$ and $\delta$ in Eq. (22) all go to zero.

\subsubsection{Integral in Vortex Sizes}

After the described simplifications the entrainment model reduces to an expression requiring only a one dimensional integral in vortex size, 


$$
\begin{aligned}
& S(D, z)=S_{\ell 0} \frac{J}{\bar{v}_{0}} p_{z}(z \mid D) p_{h}(D) \Lambda\left(\alpha_{d}\right) \\
& J=\int_{0}^{\infty} d \ell J_{\ell}(\ell) \\
& J_{\ell}(\ell)=0.8413 \frac{f_{L}}{\ell^{4}} P_{B}(\ell \mid \ell) \frac{u_{\ell}^{3} \ell^{3}}{g z_{e}} I_{Q}\left(F r_{\ell}\right)
\end{aligned}
$$

where $J\left[\mathrm{~m}^{3} / \mathrm{m}^{2} \mathrm{~s}\right]$ is the total volumetric flux of entrained air per unit area and $J_{\ell} d \ell$ represents the contribution to this flux from a vortices within $d \ell$ of size $\ell$. Each term in $J_{\ell}$ can be evaluated using Eq. (17) for $n_{\ell}$, Eq. (30) for $P_{B}$, Eq. (14) for $u_{\ell}$, Eq. (25) for $z_{e}$, and Eq. (41) for $I_{Q}$. Notice that the role of $p_{z}$ in Eq. (42) is to convert the total surface flux $J$ to a volumetric source. This conversion is done under the assumption that the upwards flux of bubbles due to buoyancy balances out the incoming flux of bubbles due to entrainment at the free surface. The vertical distribution in this pseudo equilibrium is found by assuming a given turbulence profile with depth, see $\$ 2.2 .6$ for details. In principle, a numerical method allowing to directly impose the surface flux $J$ would not need the additional modeling in $p_{z}$.

The spectral flux $J_{\ell}$ for the parameters in Table 1 is shown in Fig. 9 along with Hinze's diameter and integral length in dashed lines. Vortices much smaller than Hinze's scale do not entrain air due to the strong stabilizing effect of surface tension while large vortices with $\mathrm{Fr}_{\ell}<0.28$ do not entrain air since they only generate long wavelength waves not steep enough to trigger entrainment.

The computation of the integral in Eq. (42) is performed more accurately in a logarithmic scale. Introducing the change of variables $u=\ln (\ell)$ yields 


$$
J=\int_{-\infty}^{\infty} d u \ell J_{\ell}(\ell)
$$

which justifies the use of $\ell J_{\ell}$ in Fig. 9 instead of just the flux $J_{\ell}$. The result of this integration is shown in Fig. 10 for an air-water system. As turbulent dissipation $\varepsilon_{t}$ increases, Hinze's diameter decreases according to Eq. (15) as the smallest scales become energetic enough to entrain air. As a result the tail limited by Hinze's scale moves to the left in Fig. 9, effectively increasing the total area under the curve and therefore the total air entrainment flux. This trend is seen in Fig. 10a. The increase of turbulent kinetic energy, with a constant dissipation, has the effect of adding larger energy containing eddies and therefore the integral length scale controlled tail in Fig. 9 moves to the right effectively increasing the area under the curve. However, from Eq. (41), this increase stops for $\mathrm{Fr}_{\ell}<0.28$ since large vortices only generate long wave length waves not steep enough to entrain air, explaining the asymptotic behavior observed in Fig. $10 \mathrm{~b}$.

\subsubsection{Size Distribution}

In the final form of the model in Eq. (42), the size distribution is given in terms of the product between the history size distribution $p_{h}(D)$ and the probability of finding a bubble at a given depth $p_{z}(z \mid D)$ including buoyancy filtering. The information on the amount of entrained air, i.e. the source strength, is contained in the functional form of the total entrainment flux $J$. During the simplification process of the main framework, given by Eq. (11), it was assumed that breakup and coalescence balance each other so that the history size distribution $p_{h}(D)$ could be factored outside the model integral. In reality, entrainment is a very violent and energetic process far from equilibrium, with experiments showing large populations of bubbles with sizes under 
$50 \mu \mathrm{m}$ at the stern of a ship (Terrill and Fu 2008), which cannot be explained with the breakup processes considered here. One possible source for these small bubbles is the impact of drops trapping micrometric bubbles as observed in experiments by Pumphrey and Elmore (1990). Since this and maybe other mechanisms are not included in this work, it is not expected that the framework in Eq. (11) can provide an appropriate size distribution even if accurate models are devised for each term. In this work the focus is on deriving a model that can predict the amount of air entrainment, but using experimental data for the size distribution. Johansen et al. (2010) measured size distribution at the bow of research vessel Athena at several depths, with the closest to the free surface at a depth of $10 \mathrm{~cm}$, which is used as the entrainment size distribution $p_{e}(D)$. With this information the history size distribution is replaced by

$$
p_{h}(D)=\frac{p_{e}(D) / p_{z}(z=0 \mid D)}{\int_{0}^{\infty} d D p_{e}(D) / p_{z}(z=0 \mid D)}
$$

such that at $z=0$ the size distribution reduces to that measured by Johansen et al. (2010) or another appropriate experimental data. The entrainment size distribution allows to estimate the mean bubble volume $\bar{v}_{0}$ entrained at the free surface introduced in $\S 2.2 .1$. This is simply the mean bubble volume computed with the experimental size distribution.

\subsection{Near Free Surface Turbulence Modeling}

RANS models of turbulence are typically developed to match a series of single phase canonical flows which include shear and boundary layers and prediction of shear forces in channels and airfoils. However, to the knowledge of the authors, no corrections were developed to account for the effect of a free surface. The interaction of turbulence with a free surface is a very complex 
phenomenon involving a wide variety of scales and processes. Surface tension leads to the formation of thin liquid filaments by capillary retraction which themselves are unstable due to Rayleigh instability and break into droplets (Fuster et al. 2009). As a consequence the free surface is not a smooth continuous interface as predicted by RANS models but a very convoluted mixture of liquid filaments, droplets and bubbles mixed by turbulence. This churning two-phase flow enhances mixing by turbulence as well as the entrainment of bubbles.

These effects were found important in the simulation of the breaking wave presented in this work. While experimental observations show very high levels of turbulence at the plunging point, the blended $k-\omega / k-\varepsilon$ turbulence model is unable develop any significant turbulence given the short time scales associated with the breaking event.

To describe this additional source of randomness near the free surface, a simple mixing length model is proposed. Since no experimental or DNS simulation data are available to verify this model, the focus is to analyze trends and quantify its importance in the prediction of air entrainment and mixing. Given a mixing length $\lambda$, a velocity scale can be defined as $u_{\lambda} \approx \lambda|\boldsymbol{S}|$ with $|\boldsymbol{S}|=\sqrt{2 S_{i j} S_{i j}}$ and $S_{i j}$ the strain rate tensor. This mixing length represents the surface roughness not resolved by RANS models and is expected to be in the order of a few millimeters. A mixing length viscosity is defined by

$$
v_{\lambda}=\left(c_{\lambda} \lambda\right)^{2}|\boldsymbol{S}| \max (1-\phi / \lambda, 0)
$$

where the last term, function of the distance to the free surface $\phi$, limits the effect of this additional mixing to the neighborhood of a band with a characteristic size given by the mixing length. This additional mixing generates turbulence in the turbulent kinetic energy equation with a source given by 


$$
P_{\lambda}^{k}=2 v_{\lambda} S_{i j} \frac{\partial u_{i}}{\partial x_{j}}
$$

This model affects the flow in three important ways not considered by standard RANS models: 1) additional turbulence is created leading to an increased bubble entrainment, 2) increased turbulence mixing pushing bubbles to depth and 3) dissipation of liquid momentum that would otherwise remain in the flow for unphysically long periods of time.

To avoid the unphysical production of turbulence near stagnation points due to numerical wiggles in the velocity field greatly amplified when taking gradients, Menter (1994) proposed to use a production limiter of the form $\min \left(P_{k}, 20 \varepsilon_{t}\right)$ with $P_{k}$ the production due to shear. Though this strategy shows good agreement with experiments it lacks of any physical justification. The authors observed this limit to have a significant effect in the simulation of wave breakers and propose to remove it in the near mixing length region of the free surface, but to keep it everywhere else and in particular near the solid surfaces where it was intended to be activated.

In summary, in this work the turbulence model by Menter (1994) with modifications to bubbly flows by Moraga et al. (2008) is referred as TPM (Two Phase Menter's blended $k-\omega / k-\varepsilon$ model) while the mixing length model is referred as ML. When Menter's limit near the free surface is removed the model is referred as MNL (Menters's model with No Limit in production).

\section{Implementation Details}

The entrainment model was implemented in the dynamic overset CFD code REX, which has polydisperse bubble transport capabilities. A brief summary is discussed next, but full details 
can be found in Castro and Carrica (2013a; 2013b), Martin et al. (2015), Li et al. (2015), and references therein.

\subsection{Discretization of Bubble Sizes in Groups}

Eq. (5) is discretized following a multigroup approach (Carrica et al. 1999, Castro et al. 2013a), resulting in a transport equation for the bubble number density on each bubble size group

$$
\frac{\partial N_{g}}{\partial t}+\nabla \cdot\left(\boldsymbol{u}_{g} N_{g}\right)+F_{g+1 / 2}-F_{g-1 / 2}=\nabla \cdot\left(\frac{v_{t}}{S c_{g}} \nabla N_{g}\right)+\beta_{g}+\chi_{g}+S_{g}
$$

where the number density for group $g$ is $N_{g}(\boldsymbol{r}, t)=\int_{m_{g-1 / 2}}^{m_{g+1 / 2}} \mathrm{~d} m f(m, \boldsymbol{r}, t)$. The void fraction is then defined as the sum of all group void fractions, $\alpha_{d}(\boldsymbol{r}, t)=\sum_{g=1}^{G} \alpha_{g}=\sum_{g=1}^{G} \frac{m_{g}}{\rho_{d}\left(m_{g}, \boldsymbol{r}, t\right)} N_{g}(\boldsymbol{r}, t)$, with $G$ the number of groups. The dissolution flux is defined as $F_{g}(\boldsymbol{r}, t)=\dot{m}_{g} f\left(m_{g}, \boldsymbol{r}, t\right)$, with $\dot{m}_{g}=\dot{m}\left(m_{g}\right)$ (Castro et al. 2013b). The velocity of the dispersed phase is obtained from discretization of Eq. (6) and use of the proper models for the interfacial forces listed in Eq. (7) (Li et al. 2015),

$$
\begin{aligned}
& C_{V M}\left[\frac{\partial \mathbf{u}_{g}}{\partial t}+\left(\boldsymbol{u}_{g} \cdot \nabla\right) \boldsymbol{u}_{g}-\left(\frac{\partial \mathbf{u}_{c}}{\partial t}+\left(\boldsymbol{u}_{c} \cdot \nabla\right) \boldsymbol{u}_{c}\right)\right]+\frac{3}{8} \frac{C_{D, g}}{R_{g}}\left\|\boldsymbol{u}_{r, g}\right\| \boldsymbol{u}_{r, g}=-\nabla p_{c}+\left(\left(1-\frac{\rho_{d}}{\rho_{c}}\right) \frac{\hat{k}}{F r^{2}}\right) \\
&-C_{L} \boldsymbol{u}_{r, g} \times \nabla \times \boldsymbol{u}_{c}
\end{aligned}
$$

Discretization of the intergroup transfer terms in Eq. (47) due to dissolution $F_{g+1 / 2}-F_{g-1 / 2}$ , breakup $\beta_{g}$, and coalescence $\chi_{g}$, are key to conserve bubble mass and are treated in Castro et al. (2015a). 


\subsection{Summary of Transport Equations and Discretization}

The overall polydisperse two-fluid model is comprised of the following transport equations:

1) Continuous phase momentum equation, Eq. (1). 3 unknowns per grid point $\left(\boldsymbol{u}_{c}\right)$.

2) Mass conservation equation, Eq. (2). 1 unknown per grid point $\left(p_{c}\right)$.

3) Level set transport equation, Eq. (3). 1 unknown per grid point ( $\phi)$.

4) Turbulence model equations (Moraga et al. 2008). 2 unknowns per grid point $(k, \omega)$

5) Bubble momentum equations, Eqs. (48). $3 G$ unknowns per grid point ( $\boldsymbol{u}_{g}$ for $1<g<G$ )

6) Bubble number density equations, Eqs. (47). $G$ unknowns per grid point ( $N_{g}$ for $1<g<G$ )

In total there are 7 unknowns for the standard free surface single phase problem, plus $4 \mathrm{G}$ unknowns for the multigroup discretization of the polydisperse approach. As an example, a problem with the bubbles sizes discretized in 15 groups and solved on a 10 million point grid will have 670 million unknowns. An equivalent single-phase turbulent free surface problem will have 70 million unknowns, with variables listed in points 1) through 4) above. Though the number of unknowns is about 10 times higher, the polydisperse problem will take about 2 to 3 times more CPU time to converge, since the highest cost of the computation, the solution of the pressure equation in point (2) above, has the same cost for the single-phase or the polydisperse problems (Carrica et al. 2010).

Discretization and solution algorithms for the two-fluid polydisperse approach are complex due to the multiple non-linear coupling between the equations, and is discussed in detail in Castro et al. (2013a, 2013b) and Li et al. (2015). General discretization in REX uses multiblock structured curvilinear body-fitted grids with a dynamic overset capability that allows the placement of local 
refinements where needed and to perform computations with large amplitude motions. A coordinate transformation from the physical domain to the computational domain is used to discretize the equations, where control volumes in the computational domain are cubes with unit volume and area on each face. The governing equations are discretized using a control volume/finite differences approach on a collocated grid in space. The convection terms are discretized with linear upwind biased or TVD schemes up to fourth order accurate in space (Ismail et al. 2010). Diffusion terms use a second-order central discretization while temporal terms are discretized using a second-order backwards scheme. Suggar is used to dynamically obtain overset interpolation coefficients (Carrica et al. 2010). Pressure-velocity coupling is achieved using a hybrid projection approach as discussed in Li et al. (2015).

\section{Model Evaluation and Applications}

Simulations were performed to evaluate the performance of the model against available experimental data. The first case consists of a breaking wave. Though the proposed entrainment model is designed for turbulent entrainment as the main process, the mixing length turbulence model in the neighborhood of the free surface can reasonably well describe the large random fluctuations representing free surface fragmentation, leading to air entrainment predictions in good agreement with the experiments. The second case is a full scale simulation of the research vessel Athena to test the model's performance in problems of engineering interest involving free surface flows around complex solid geometries. A grid study is performed demonstrating the grid independence of the model. Predictions are compared with experimental data qualitatively and quantitatively showing promising results relevant to ship hydrodynamics. 


\subsection{D+T Breaking Wave}

Tavakolinejad (2010) performed a set of experiments to simulate the breaking bow wave crated by an advancing ship using a $2 \mathrm{D}+\mathrm{T}$ technique. Driven by a series of pistons a steel board deforms and pushes water in a tank to model the shape of a ship as it crosses with speed $U$ a fixed plane in space. If three dimensional effects are ignored, the resulting traveling wave at a time $t$ mimics a slice transverse to the ship at a position $x=t U_{0}$. Experimental results are therefore reported in terms of longitudinal location along this virtual ship when in reality refers to a particular point in time. The same convention is followed here.

The experiment simulated the DTMB 5415 surface combatant model without sonar bulb with a waterline length $L=21.03 \mathrm{~m}$ moving at the speed $U=5.441 \mathrm{~m} / \mathrm{s}$, resulting in Froude and Reynolds numbers of $\mathrm{Fr}=0.379$ and $R e=1.14 \times 10^{8}$, respectively. The Tank used in the experiment was $14.8 \mathrm{~m}$ long, $1.15 \mathrm{~m}$ wide, and $2.2 \mathrm{~m}$ deep.

The simulation is performed in $2 \mathrm{D}$ as shown in Fig. 11. To retain a proper level of refinement for the plunging breaker and the regions of interest where bubbles are formed and transported, three overset blocks shown in Fig. 11 are designed. Block 1 (68 K grid nodes) discretizes the large wave tank used in the experiment. The left side of the domain follows the wave board shape in time by linearly interpolating the profiles reported in the experiment. To maintain good grid quality throughout the board deformation, the inner grid points of Block 1 are moved using linear interpolation between 13 pre-generated grids together with smoothing. Block 3 (234 K grid nodes with $\Delta \cong 4 \mathrm{~mm}$ ) consists of a Cartesian grid that rigidly moves in time to follow the main features of the wave created and the clouds of bubbles entrained. Block $2(221 \mathrm{~K}$ grid nodes with $\Delta \cong 0.7 \mathrm{~mm}$ ) consists of a smaller Cartesian grid that closely follows the position 
of the breaker to properly refine and capture the plunge and subsequent cavity formation and collapse. The trajectory of the refinement blocks following the main features of interest is estimated from an initial single phase run without bubbles.

The surface elevation at the wave board is compared against experimental results in Fig. 12, where the elevation is non-dimensionalized with the ship's draft $T=0.914 \mathrm{~m}$ and the time with the wavemaker runtime $T_{w m}=1.93 \mathrm{~s}$. The peak elevation is well predicted and good agreement is observed, though the computed rise and fall times are slightly longer than in the experiment.

The air entrainment constant in Eq. (22) is first estimated in $S_{\ell 0}=2.6 \times 10^{-2}$ by matching void fraction levels with the available experimental data at the stern of the research vessel Athena (Johansen et al., 2010). Air entrainment in this region is caused by the strong turbulence in the wake of the ship where the modeling assumptions used for the entrainment model are expected to work best. Further, the $2 \mathrm{D}+\mathrm{T}$ breaker is run with a coarse discretization in bubble size of four groups for a quick estimation of the mixing length model constants. The air entrainment size distribution $p_{e}(D)$ in Eq. (44) matches the measurements at the bow of Athena R/V (Johansen et al., 2010).

From these initial runs the mixing length model constant in Eq. (45) is calibrated to $c_{\lambda}=0.03$. Measurements during the acoustic phase of a breaker lead Deane and Stokes (2002) to estimate levels of turbulent dissipation in the order of $\varepsilon_{t}=13 \mathrm{~m}^{2} / \mathrm{s}^{3}$, while numerical simulations performed by Moraga et al. (2008) predict dissipations in the range $\varepsilon_{t}=29-68 \mathrm{~m}^{2} / \mathrm{s}^{3}$, though for stronger breakers. With the calibrated mixing length model constant, the simulations of the $2 \mathrm{D}+\mathrm{T}$ 
breaker predict dissipations at the impact point in the range $4<\varepsilon_{t}<100 \mathrm{~m}^{2} / \mathrm{s}^{3}$, in good agreement with those authors' investigations.

After these initial calibration runs, simulations are performed using 15 bubble groups logarithmically distributed from $20 \mu \mathrm{m}$ to $1 \mathrm{~cm}$ in diameter to better describe breakup, coalescence and filtering due to buoyancy. The time step is set to $\Delta t=5.4 \times 10^{-4} \mathrm{~s}$.

Figure 13 shows void fraction at $x / L=0.5$ predicted using the three turbulence models discussed in $§ 2.4$, compared with a photograph from experiments by Tavakolinejad (2010). Two bubble clouds are expected at this stage; a first cloud due to the main plunging breaker and a second cloud due to a secondary spilling breaker. The TPM model fails to predict a strong first breaker. This is caused by Menter's limit in turbulent production that is particularly important for this fast developing turbulent free surface flow. The prediction is greatly improved when the limit is removed in the TPM+MNL model. However, the void fraction is underpredicted for the first breaker and overpredicted for the second breaker, due to an excess of mechanical energy. When using the mixing length model more turbulence, and therefore entrainment, is produced during the first breaking event while energy is dissipated from the flow and the resulting second breaker is smaller matching better the experimental results. The TPM+MNL+ML model shows good qualitative agreement with the experimental picture shown in Fig. 13 and is therefore used in the quantitative studies that follow.

The void fraction of the entrained bubbles as the breaking wave is formed and evolves in two bubble clouds is shown in Fig. 14 and in the animated version provided in Electronic Annex 1. This figure compares qualitatively very well with the results of Derakhti and Kirby (2014), who simulated air entrainment by a breaking wave using a highly resolved 3D volume of fluid method 
capturing the largest air cavities and modeling under-resolved bubble entrainment with a model based on the work of Ma et al. (2011a). As in the case of Derakhti and Kirby (2014), simulations show a second bubble cloud with higher void fraction reaching as much as $30 \%$. An incipient third cloud in Fig 14, also present in Derakhti and Kirby (2014) simulations, is formed by a spilling wave dissipating the remaining energy of the wave.

Figure 15 shows experimental void fraction as a function of depth for each bubble cloud as reported by Tavakolinejad (2010). Averaging is performed across the cloud at constant depth, to mimic the procedure followed in the experiments.

Experiments show that clouds can reach $20 \mathrm{~cm}$ in depth at $x / L=0.5$ and grow to depths of $30 \mathrm{~cm}$ at $x / L=1$ as they mix by turbulence. The void fraction decreases from $x / L=0.5$ to $x / L=1$ due to this expansion, but mostly as bubbles rise and escape through the free surface. A similar trend is observed in the simulations though bubbles do no reach as deep as in the experiment. Air entrainment and consequently the void fraction predictions are in fairly good agreement with the experiments for the four locations shown, with the exception of the second cloud at $x / L=0.5$, where the entrainment model overpredicts the void fraction by one order of magnitude. This is due to entrainment lasting longer in CFD for the second splash up that causes the second cloud, a trend also observed in the wave of Derakhti and Kirby (2014). At $x / L=1$ most bubbles leave the domain due to buoyancy and results are again comparable with experiments for both clouds.

Number density distributions are reported in Fig. 16 for experiments and CFD, averaged over the whole cloud. The slope of the profile for bubbles smaller than about $300 \mu \mathrm{m}$ in diameter shows good agreement with the experimental data. For bubbles larger than $300 \mu \mathrm{m}$ the 
experiments seem to exhibit a bulge not present in the CFD predictions. This is more evident in Fig. 17, which shows normalized void fraction distribution where a bimodal shape is observed with peaks at around $150 \mu \mathrm{m}$ and $800 \mu \mathrm{m}$ that the CFD model does not predict. The normalized void fraction distribution $\bar{\alpha}_{D}(D)$ in Fig. 17 is defined such that the area under each curve in this logarithmic scale is one, i.e. $\int_{0}^{\infty} \bar{\alpha}_{D}(D) d(\ln (D))=1$.

Figures 16 and 17 suggest that the entrainment size distribution used in the model may be lacking very large bubbles, or cavities, that quickly leave the domain due to buoyancy. Notice in Fig. 14 that the entrainment phase in the evolution of the second bubble cloud is still active at $x / L=0.4$, where the void fraction is approximately $30 \%$. As the second cloud reaches $x / L=0.5$ the void fraction decreases by one order of magnitude, but still over predicts the experimental void fraction data by one order of magnitude. Though large bubbles are present in the entrainment size distribution (see Fig. 16), very large air cavities (bigger than $5 \sim 10 \mathrm{~mm}$ in diameter) present during the wave breaking process observed in the experiments are missing in the ocean size distribution from Johansen et al. (2010) that is used in the entrainment model. Still, considering the complexity of the problem, the bubble entrainment model can predict the main features of the two-phase flow.

Though differences between experiments and computations are larger for the second cloud, the first cloud could see better results. A series of factors, related to other closure models used on the overall solution strategy, affect the bubble entrainment. One of them is coalescence in the presence of surfactants, of significance in salt water. The Prince and Blanch (1990) model is used for coalescence. It is well known that coalesce is inhibited in the presence of electrolytes, and Lessard \& Zieminski (1971) report a coalescence efficiency of only 35\% in salt water compared to that of fresh water at 21 degrees Celsius. To account for this inhibition we use a 0.35 factor 
multiplying the coalescence kernel of Prince and Blanch (1990) for all of our computations involving seawater (or Instant Ocean salt mix in the case of Tavakolinejad (2010) experiments). This constant factor assumes the coalescence inhibition to be a function independent of bubble size. This is discussed with great detail in Castro et al. (2013a) where a functional dependence with bubble size is proposed. However we found the simple constant factor of $35 \%$ (which in addition is based on reported experimental data) to be more appropriate.

Also of great importance is the turbulence closure model, which in this case is an unsteady RANS approach. A Large Eddy Simulation (LES) or hybrid RANS/LES would result in considerably more resolved turbulent structures, and likely transport of bubbles deeper as the bubbles develop. Use of LES approaches would require a 3D grid, with the consequent additional cost, and probably a new calibration of constants appropriate for LES, since the resolved turbulence will change. This is planned as future work.

\subsection{Bubbly Flow Around Athena $R / V$}

The US Navy research vessel Athena is a decommissioned PG-84 Asheville-class patrol gunboat transformed into a high-speed research vessel in 1976. The ship is $47 \mathrm{~m}$ long and can run on diesel engines up to 14 knots and on gas turbines at speeds in excess of 35 knots. Athena R/V offers a good opportunity to test the entrainment model in a relevant full-scale geometry where data is available. Johansen et al. (2010) measured void fraction and bubble size distribution at the bow, masker and stern for speeds ranging from 3.1 to $6.2 \mathrm{~m} / \mathrm{s}$ ( 6 to $12 \mathrm{knots}$ ).

The overset grid system, shown in Fig. 18, is designed to resolve the flow around the fully appended ship, the bow breaking wave, and the highly energetic flow in the transom stern. Refinements blocks shown as colored boxes in Fig. 18 are placed at bow, masker, and stern regions 
as required by the entrainment model. As the focus of this simulation is to evaluate the entrainment model occurring near the free surface far from propellers, a body force model using the radial load distribution from Hough and Ordway (1964) is used, allowing larger time steps and the use of a static overset grid system. Though Fig. 18 shows the full ship for clarity, symmetry about the centerplane is considered to reduce grid size and computational cost.

To test grid convergence, a critical and desirable property of the entrainment model, a grid refinement study is performed with five grids: Very Coarse (VCoarse), Coarse, Medium, Fine, and Very Fine (VFine). The refinement ratio in each direction is $\sqrt[3]{2}$, doubling the total number of grid cells with each successive refinement. Table 2 shows details of the different grids, with $d x$ the grid spacing in the streamwise direction.

The simulations are performed at a speed of $U=5.4 \mathrm{~m} / \mathrm{s}$ (10.5 knots), resulting in Froude and Reynolds numbers of 0.252 and $2.53 \times 10^{8}$, respectively. A regular incoming wave with amplitude is $0.05 \mathrm{~m}$ and wave length $35.2 \mathrm{~m}$ is applied at the inlet boundary, mimicking the experimental conditions of Johansen et al. (2010). A small time step of $\Delta t=9.2 \mathrm{~ms}$ is used to avoid excessively large Courant numbers for all the grid systems.

The constants for the air entrainment and mixing length turbulence models are the same as for the $2 \mathrm{D}+\mathrm{T}$ bow breaking wave case presented in the previous section. The simulation is performed for three ship lengths until a periodic solution driven by the incoming waves is achieved. Two additional wave periods are then simulated to perform averages on the solutions. Electronic Annex 2 shows an animation of the free surface colored by wave height on port and by void fraction on starboard that allows to appreciate the periodic entrainment caused by the incoming waves. 
Figure 19 shows void fraction contours on the free surface for all the grids. The black dots inside the figure indicate the positions where solutions are extracted at different depths for comparison with experimental data. Bubbles entrained at the breaking bow wave region are transported downstream and eventually mix with the bubbly flow at the stern of the ship. The Kelvin waves are captured more clearly as the grid is refined. Qualitatively, the mean void fraction at the stern seems to converge in grid, and a quantitative analysis is provided in the following sections. The bow insets on the left of Fig. 19 show details of the void fraction contours and exhibit grid convergence of the solution in this region. Figure 20 shows void fraction contours on the hull and the free surface. An animated version is provided in Electronic Annex 3. Significant entrainment is observed in the contact line between free surface and hull, being this a highly turbulent region. In addition, bubbles entrained at the bow are swept down underneath the ship by convective transport and turbulent mixing. Bubbles entrained at the bow are swept down by the flow forming a characteristic trace of higher void fraction all along the hull that finally is forced to flow between the struts by the suction of the propellers. These bubbles have the potential to interact with the strongly changing pressure field across the propellers' planes leading to an increased aeration by rectified diffusion (Raju et al., 2009).

Overall, the void fraction contours on the hull show qualitative convergence and no major difference can be observed between the finest grids. A formal convergence study was performed for the total average entrainment source at the transom of Athena $\mathrm{R} / \mathrm{V}$, where the entrainment is strictly due to turbulence/free surface interaction. The total source is computed as

$$
S_{\text {int }}=\left\langle\underset{V}{\underset{\mathrm{O}}{\mathrm{O}}} \underset{0}{¥} S(D, z) v_{D} d D d V\right\rangle
$$


where $V$ is the region delimited by $(1.05 \leq x \leq 1.18,0 \leq y \leq 0.045,-0.01 \leq z \leq 0.01)$, chosen to include the entire region where entrainment is active at the stern, $v_{D}$ is the volume for a bubble with diameter $D$, and \langle\rangle performs the time average operation in two wave periods. Table 3 shows the resulting total bubble entrainment source $S_{\text {int }}$ for all grids as a function of the relative refinement $\mathrm{D} x / \mathrm{D} x_{0}$, where $\mathrm{D} x_{0}$ is the reference discretization size for the VCoarse grid. The total air entrainment rate at the stern considering both sides of the ship at the given operational condition corresponds to $400 \mathrm{~m}^{3} / \mathrm{s}$ in dimensional terms. There are three possible sets of grids using a $\sqrt[3]{2}$ refinement ratio (VCoarse, Coarse, Medium), (Coarse, Medium, Fine) and (Medium, Fine, VFine), and one set with refinement ratio $2^{2 / 3}$ (VCoarse, Medium, VFine). Figure 21 shows the total source as a function of the relative refinement. Notice that as the grids are refined the entrainment source converges, except for the VCoarse grid which does not follow the same trend as the other grids, evidence that the VCoarse grid is not fine enough to be in the asymptotic solution range. Following the procedure of Stern et al. (2001), the summary of the grid study is presented in Table 4. The set including the VCoarse, Medium and VFine grids shows that the grids are not in the asymptotic range, since the correction factor $C_{G}$ is significantly smaller than one. This set also shows very low accuracy $\left(P_{G}=1.08\right)$ and large grid error and uncertainty $\left(\delta_{G}^{*}=1.93\right.$ and $U_{G}=1.71 \%$ of the VFine grid value, respectively). When the four finest grids are used, the order of accuracy increases to $P_{G}=2.23$ for the three intermediate grids and $P_{G}=1.82$ for the three finest grids, more in line with the expected order of accuracy since except for convection, all other terms use second-order discretization in space. Notice also in electronic Annex III that the VCoarse grid exhibits different behavior on the shaft/struts/rudder region, too coarse to capture separation 
form the appendages. This, again, is consistent with the grid study that showed that using the VCoarse grid did not yield to asymptotic behavior.

\subsubsection{Void fraction profiles}

Void fraction profiles with depth are shown in Fig. 22. Simulation results are sampled at $(x, y)=(0.158,0.052)$, or about 3.5 meters downstream of the second bubble cloud at the bow, while in the experiments the probe was located approximately 3.5 meters downstream of the bow wave breaker. Although grid convergence is not evident, the difference among the five grids is small considering how sensitive the breakers are to small changes in turbulence levels. The void fraction measurements at that location are not reliable, since in the experiments ship motions were a problem, with roll pitch and heave causing the probe to come periodically out of the water. Johansen et al. (2010) processed their raw data by filtering the signals when the probe was in air for too long, which was deemed as an 'out of the water' event, and therefore the time the probe is out of the water is not counted as additional void fraction. This, however, is acknowledged by the authors as a significant source of error in the experiment that can lead to considerable overprediction of the void fraction since the probe frequently was exposed to air and the cutoff chosen for filtering changed the void fraction considerably. The experimental bubble size distributions are deemed reliable, since the 'out of water' events that are counted affect greatly the void fraction but add only very large bubbles to the size distributions, which were eliminated from the final reported size distribution. The void fraction is under predicted by about one order of magnitude compared with experimental data and therefore two axes are used in Fig. 22.

Void fraction profiles with depth at the stern are shown in Fig. 23. The location for the experimental position at the stern is $(x, y)=(1.0106,0.021)$ (Johansen et al., 2010). Although the 
experimental data from Terrill and Fu (2008) was taken at the centerline and 0.2 meters aft of Johansen et al. (2010) probe location and no filtering was performed to account for when the probe is out of the water, it is included here for completeness and because of the scarcity of experimental results. Generally, good agreement is found between the predicted profiles and the experiments even for the VCoarse grid at depths lower than around $0.5 \mathrm{~m}$. Below the transom the void fraction is very small and not visible in the scale of Fig. 23. In this region the experiments show higher void fraction levels than those obtained in the simulation, possibly due to underprediction of bubbles coming from underneath the ship or production of bubbles by the propeller by rectified diffusion (Raju et al., 2009).

Overall, void fraction shows good convergence in depth though it is not monotonically convergent near the free surface. The differences in void fraction among the five grids are below $3 \%$, which is remarkable for an entrainment model at the stern's highly ventilated flow.

\subsubsection{Bubble size distribution}

Normalized bubble size distributions for several depths at the stern are reported in Fig. 24. The bubble groups reported in the experiment (Johansen et al., 2010) are different from those used in the numerical simulations, thus the numerical results are integrated over the experimental groups for comparison. At $0.1 \mathrm{~m}$ deep the profiles for the five grids are practically indistinguishable from each other as a result of the entrainment source being the dominating term at this depth. The profiles show convergence at depths of $0.3 \mathrm{~m}$ and $0.5 \mathrm{~m}$. However, a significant difference among grids is observed at $0.6 \mathrm{~m}$, where a sharp transition in the turbulence field occurs as the slow speed recirculating transom flow transitions into the high speed boundary layer flow coming from below the ship. Although the VCoarse grid predicts large bubbles dominating the size distribution, small 
bubbles become the main contribution as the grid is refined. The reason for such large difference is the difficulty in predicting the sharp transition with the coarse grid, and numerical diffusion strongly affects the size distribution around this region. As depth increases further, the profiles show good grid convergence. Overall, the normalized group void fraction shows very good grid convergence trends.

The experimental data exhibits two peaks at all depths in Fig. 24 These peaks are not always predicted by the numerical simulation. At $0.1 \mathrm{~m}$, the experimental data exhibits a small peak at $R=80 \mu \mathrm{m}$ not predicted by the two phase model which at that location is dominated by the entrainment size distribution. This peak is also seen at all other depths in the experiments suggesting that a fraction of these bubbles might come from the ship's boundary layer. Another fraction can be entrained form the surface by droplet impact, a process important in the highly energetic stern flow and ignored at this point in the entrainment model. A third possibility is that some of the small bubbles are the product of breakup of large bubbles. The experimental data shows a peak at $R=2 \mathrm{~mm}$ at $0.3 \mathrm{~m}$, underpredicted slightly by the simulations. At $0.6 \mathrm{~m}$, the numerical simulation predicts a peak at around $R=80 \mu \mathrm{m}$, present in the experimental data, and repeated for depths $0.6 \mathrm{~m}, 0.65 \mathrm{~m}$ and $0.7 \mathrm{~m}$.

\section{Conclusions}

A mechanistic model of bubble entrainment due to turbulence/free surface interaction is presented. The model is based on a framework that splits the entrainment into separate fundamental processes, from bubble creation by vortex/free surface interaction, to transport to depth as bubbles coalesce and break up. Models for the individual processes are derived to arrive to a closed 
entrainment model. The final model was implemented in the overset free surface code REX and tested for two cases with experimental data. The model constants are calibrated once and used to predict bubble entrainment for two very different entrainment scenarios with promising results.

Comparisons of model performance against experimental results for a breaking wave show that the model can predict entrainment location and magnitude well, though the void fraction is over predicted for the second bubble cloud produced by the experiment near the breaking point. As the bubble clouds evolve the predictions compare better with the experiments. The predicted bubble size distributions are close to those measured in the experiments, but the influence of the entrainment size distribution, taken from experimental data on a full scale ship in this work, can dramatically affect the predicted size distribution. At this time the entrainment size distribution is not modeled but imposed, and producing a model for this important quantity is left as future work.

For the first time a grid study was performed to test the ability of an entrainment model to converge in grid, showing good results. Satisfying this important property for entrainment models has been a concern in the community because performing grid studies is very expensive for already costly polydisperse computations.

In addition to already mentioned work on entrainment size distribution modeling, future work will concentrate on refinement of the individual models for the different mechanisms leading to entrainment, as well as further validation and test against canonical flows without solid boundaries like a hydraulic jump, and for a flat bottom boat with extensive experimental data (Perret and Carrica, 2015). 


\section{Acknowledgements}

This work was supported by the Office of Naval Research grant N00014-14-1-0134 with

Drs. Patrick Purtell, Ki-Han Kim and Thomas $\mathrm{Fu}$ as program officers. Computations were performed on the Cray XC30 (Lightning), at the U.S. Air Force Research Laboratory Shared Resource Center.

\section{References}

Anthony, D.G., Willmarth, W.W., 1992. Turbulence measurements in a round jet beneath a free surface. J. Fluid Mech. 243, 699-720.

Assirelli, M., Bujalski, W., Eaglesham, A., Nienow, A.W., 2008. Macro-and micromixing studies in an unbaffled vessel agitated by a Rushton turbine. Chem. Eng. Sci. 63, 35-46.

Baddour, R.E., Zaghloul, A., Martunizzi, R., 2006. Entrainment properties of plane surface jets in shallow current. J. Hydr. Eng., ASCE 132, 363-370.

Baldy, S., 1993. A generation-dispersion model of ambient and transient bubbles in the close vicinity of breaking waves. J. Geophys. Res.: Oceans 98, 18277-18293.

Behzadi, A., Issa, R.I., Rusche, H., 2004. Modelling of Dispersed Bubble and Droplet Flow at High Phase Fractions. Chem. Eng. Sci. 59, 759-770.

Biń, A.K., 1993. Gas entrainment by plunging liquid jets. Chem. Eng. Sci. 48, 3585-3630.

Bonetto, F., Lahey, R.T., 1993. An experimental study on air carryunder due to a plunging liquid jet. Int. J. Multiphase Flow 19, 281-294.

Callaghan, A.H., Stokes, M.D., Deane, G.B., 2014. The effect of water temperature on air entrainment, bubble plumes, and surface foam in a laboratory breaking-wave analog. J. Geophys. Res.: Oceans 119, 7463-7482.

Carrica, P.M., Bonetto, F., Drew, D., Lahey, R.T., 1999. A Polydisperse Model for Bubbly TwoPhase Flow Around a Surface Ship. Int. J. Multiphase Flow 25, 257-305.

Carrica, P.M., Wilson, R.V., Stern, F., 2007. An unsteady single-phase level set method for viscous free surface flows. Int. J. Num. Meth. Fluids 53, 229-256.

Carrica, P.M., Huang, J., Noack, R., Kaushik, D., Smith, B., Stern, F., 2010. “Large-Scale DES Computations of the Forward Speed Diffraction and Pitch and Heave Problems for a Surface Combatant," Comput. Fluids 39, 1095-1111.

Carrica, P.M., Castro, A.M., Li, J., Politano, M., Hyman, M., 2012. Towards an air entrainment model. $29^{\text {th }}$ Symp. Naval Hydrodynamics, Gothenburg, Sweden. 
Castro, A.M., Carrica, P.M., 2013a. Bubble size distribution prediction for large-scale ship flows: model evaluation and numerical issues. Int. J. Multiphase Flow 57, 131-150.

Castro, A.M., Carrica, P.M., 2013b. Eulerian polydispersed modeling of bubbly flows around ships with application to Athena R/V. Int. Shipbuilding Progr. 60, 403-433.

Chen, D., Jirka, G.H., 1999. LIF study of plane jet bounded in shallow water layer. J. Hydr. Eng., ASCE 125, 817-826.

Chen, P., Duduković, M.P., Sanyal, J., 2005. Three-dimensional simulation of bubble column flows with bubble coalescence and breakup. AIChE J. 51, 696-712.

Cox, D.T., Shin, S., 2003. Laboratory measurements of void fraction and turbulence in the bore region of surf zone waves. J. Eng. Mech. 129, 1197-1205.

Deane, G.B., Stokes, M.D., 1999. Air entrainment processes and bubble size distributions in the surf zone. J. Phys. Oceanogr. 29, 1393-1403.

Deane, G.B., Stokes, M.D., 2002. Scale dependence of bubble creation mechanisms in breaking waves. Nature 418, 839-844.

Derakhti, M., Kirby, J. T., 2014. Bubble entrainment and liquid-bubble interaction under unsteady breaking waves. J. Fluid Mech., 761, 464-506.

Dommermuth, D.G., Lewis, C.D., Tran, V.H., Valenciano, M.A., 2014. Direct simulations of wind-driven breaking ocean waves with data assimilation. In: $30^{\text {th }}$ Symp. Naval Hydrodynamics, Hobart, Tasmania, Australia.

Drew, D.A., Passman, S.L., 1999. Theory of Multicomponent Fluids, Springer, New York.

Duncan, J.H., 2001. Spillng breakers. Annu. Rev. Fluid Mech. 33, 519-547.

Ervine, D., McKeogh, E., Elsawy, E., 1980. Effect of turbulence intensity on rate of air entrainment by plunging water jets. In: ICE procs. $69,425-445$.

Ervine, D.A., 1998. Air entrainment in hydraulic structures: A review. In: Proc. of the ICE-Water Maritime and Energy 130, 142-153.

Fuster, D., Agbaglah, G., Josserand, C., Popinet, S., Zaleski, S., 2009. Numerical simulation of droplets, bubbles and waves: state of the art. Fluid Dyn. Res. 41, 065001.

Garrett, C., Li, M., Farmer, D., 2000. The connection between bubble size spectra and energy dissipation rates in the upper ocean. J. Phys. Oceanogr. 30, 2163-2171.

Hinze, J.O., 1955. Fundamentals of the hydrodynamic mechanism of splitting in dispersion processes. AIChE J. 1, 289-295.

Hough, G.R., Ordway, D.E., 1964. The generalized actuator disk. Technical Report TAR-TR 6401, Therm. Advanced Research Inc.

Hunt, J.C.R, 1984. Turbulence structure and turbulent diffusion near gas-liquid interfaces. In: Gas Transfer at Water Surfaces, 67-82.

Ishii, M., 1975. Thermo-fluid dynamic theory of two-phase flow, Collection de la Direction des études et recherches d'Électricité de France, Eyrolles. 
Ismail, F., Carrica, P.M., Xing, T., Stern, F., 2010. "Evaluation of Linear and Non-Linear Convection Schemes on Multidimensional Non-Orthogonal Grids with Applications to KVLCC2 Tanker," Int. J. Num. Meth. Fluids 64, 850-886.

Johansen, J.P, Castro, A.M., Carrica, P.M., 2010. Full-scale two-phase flow measurements on Athena research vessel. Int. J. Multiphase Flow 36, 720-737.

Kuboi, R., Komasawa, I., Otake, T., 1972. Behavior of dispersed particles in turbulent liquid flow. J. Chem. Eng. Japan 5, 349-355.

Kumagai, I., Takahashi, Y., Murai, Y., 2015. Power-saving device for air bubble generation using a hydrofoil to reduce ship drag: Theory, experiments, and application to ships. Ocean Eng. 95, 183-194.

Lehr, F., Millies, M., Mewes, D., 2002. Bubble-size distributions and flow fields in bubble columns. AIChE J. 48, 2426-2443.

Lessard, R.R., Zieminski, S.A., 1971. Bubble Coalescence and Gas Transfer in Aqueous Electrolytic Solutions. Ind. Eng. Chem. Fundam. 10, 260-268.

Levy, R., Uminsky D., Park, A., Calambokidis, J., 2011. A theory for the hydrodynamic origin of whale flukeprints. Int. J. Nonlinear Mech. 46, 616-626.

Lewis, D.A., Davidson, J.F., 1982. Bubble splitting in shear flow. Trans. Inst. Chem. Eng. 60, 283291.

Li, J., Castro, A.M., Carrica, P.M., 2015. A pressure-velocity coupling approach for high-void fraction free surface bubbly flows. Int. J. Num. Meth. Fluids 79, 343-369.

Ling, Y., Zaleski, S., Scardovelli, R., 2015. Multiscale simulation of atomization with small droplets represented by a Lagrangian point-particle model. Int. J. Multiphase Flow 76, 122143.

Lubin, P., Glockner, S., 2015. Numerical simulations of three-dimensional plunging breaking waves: generation and evolution of aerated vortex filaments. J. Fluid Mech., 767, 364-393.

Luo, H., Svendsen, H.F., 1996. Theoretical model for drop and bubble breakup in turbulent dispersions. AIChE J. 42, 1225-1233.

Ma, J., Oberai, A.A., Drew, D.A., Lahey, R.T., Moraga, F.J., 2010. A quantitative sub-grid air entrainment model for bubbly flows-plunging jets. Comput. Fluids 39, 77-86.

Ma, G., Shi, F., Kirby, J.T., 2011a. A polydisperse two-fluid model for surf zone bubble simulation. J. Geophys. Res.: Oceans, 116, C05010.

Ma, J., Oberai, A., Hyman, M., Lahey, R. T. Jr., Drew, D., 2011b. Two-fluid modeling of bubbly flows around surface ships using a phenomenological subgrid air entrainment model. Comput. Fluids 52, 50-57.

Mann, J., 1994. The spatial structure of neutral atmospheric surface-layer turbulence. J. Fluid Mech. 273, 141-168.

Martin, J.E., Michael, T.J., Carrica, P.M., 2015. Submarine Maneuvers Using Direct Overset Simulation of Appendages and Propeller and Coupled CFD/Potential Flow Propeller Solver. J. Ship Res. 59, 31-48. 
Martínez-Bazán, C., Montañés, J.L., Lasheras, J.C., 1999. On the breakup of an air bubble injected into a fully developed turbulent flow. Part 1. Breakup frequency. J. Fluid Mech. 401, 157182.

Menter, F.R., 1994. Two-equation eddy-viscosity turbulence models for engineering applications. AIAA J. 32, 1598.

Moraga, F.J., Carrica, P.M., Drew, D.A., Lahey, R.T., 2008. A sub-grid air entrainment model for breaking bow waves and naval surface ships. Comput. Fluids 37, 281-298.

Mori, N., Suzuki, T., Kakuno, S., 2007. Experimental study of air bubbles and turbulence characteristics in the surf zone. J. Geophys. Res.: Oceans 112, C05014.

Odgaard, A.J., 1986. Free-surface air core vortex. J. Hydr. Eng., ASCE 112, 610-620.

Patwardhan, A.W., Mali, R.G., Jadhao, S.B., Bhor, K.D., Padmakumar, G., Vaidyanathan, G., 2012. Argon entrainment into liquid sodium in fast breeder reactor. Nucl. Eng. Des. 249, 204-211.

Perret, M., Carrica, P.M., 2015. Bubble-Wall Interaction and Two-Phase Flow Parameters on a Full-Scale Boat Boundary Layer. Int. J. Multiphase Flow 73, 289-308.

Politano, M., Carrica, P.M., Weber, L., 2009. A multiphase model for the hydrodynamics and total dissolved gas in tailraces. Int. J. Multiphase Flow 35, 1036-1050.

Pope, S.B., 2000. Turbulent Flows. Cambridge University Press.

Prince, M.J., Blanch, H.W., 1990. Bubble coalescence and break-up in air-sparged bubble columns. AlChE J. 36, 1485-1499.

Pumphrey, H.C., Elmore, P.A., 1990. The entrainment of bubbles by drop impacts. J. Fluid Mech. 220, 539-567.

Raju, R., Hsiao, C.-T., Chahine, G. L., 2009. Influence of propeller presence and cavitation on liquid nuclei population. CAV2009 - $7^{\text {th }}$ International Symposium on Cavitation, Ann Arbor, MI, USA.

Rice, R.G., 2006. Analytical solutions for film thinning dynamics in bubble coalescence. AIChE J. 52, 1621-1622.

Rousseaux, J.M., Muhr, H., Plasari, E., 2000. Chemical reactors of special geometry for the precipitation of mineral particles. Can. J. Chem. Eng. 78, 650-662.

Shi, F., Kirby, J.T., Ma, G., 2010. Modeling quiescent phase transport of air bubbles induced by breaking waves. Ocean Modelling 35, 105-117.

Souders, D.T., Hirt, C.W., 2004. Modeling entrainment of air at turbulent free surfaces. Proceedings of World Water and Environmental Resources Congress, 1-10.

Stern, F., Wilson, R.V., Coleman, H.V., Paterson, E.G., 2001. Comprehensive approach to verification and validation of CFD simulations-Part 1\&2: methodology and procedures, ASME J. Fluids Eng. 124, 793-802.

Tavakolinejad, M., 2010. Air bubble entrainment by breaking bow waves simulated by a 2D+T technique. PhD thesis, The University of Maryland. 
Terrill, E.J., Fu, T., 2008. At-sea measurements for ship hydromechanics. In: $27^{\text {th }}$ Symp. Naval Hydrodynamics, Seoul, Korea.

Walker, D.T., Johnson, V.G., 1991. Observations of turbulence near the free surface in the wake of a model ship. In: Dynamics of bubbles and vortices near a free surface, ASME AMD119.

Wang, T., Wang, J., Jin, Y., 2003. A novel theoretical breakup kernel function for bubbles/droplets in a turbulent flow. Chem. Eng. Sci. 58, 4629-4637.

Wang, Z., Yang, J., Stern, F., 2014. High-fidelity simulations of bubble, droplet, and spray formation in breaking waves. $30^{\text {th }}$ Symp. on Naval Hydrodynamics, Hobart, Tasmania, Australia.

Yue, Y., Green, N.R., 2012. Modelling of different entrainment mechanisms and their influences on the mechanical reliability of Al-Si castings. In: IOP Conference Series: Materials Science and Engineering 33, 012072.

Zhang, L., Thomas, B.G., 2003. State of the Art in Evaluation and Control of Steel Cleanliness. ISIJ Int. 43, 271-291. 


\section{Main Symbols}

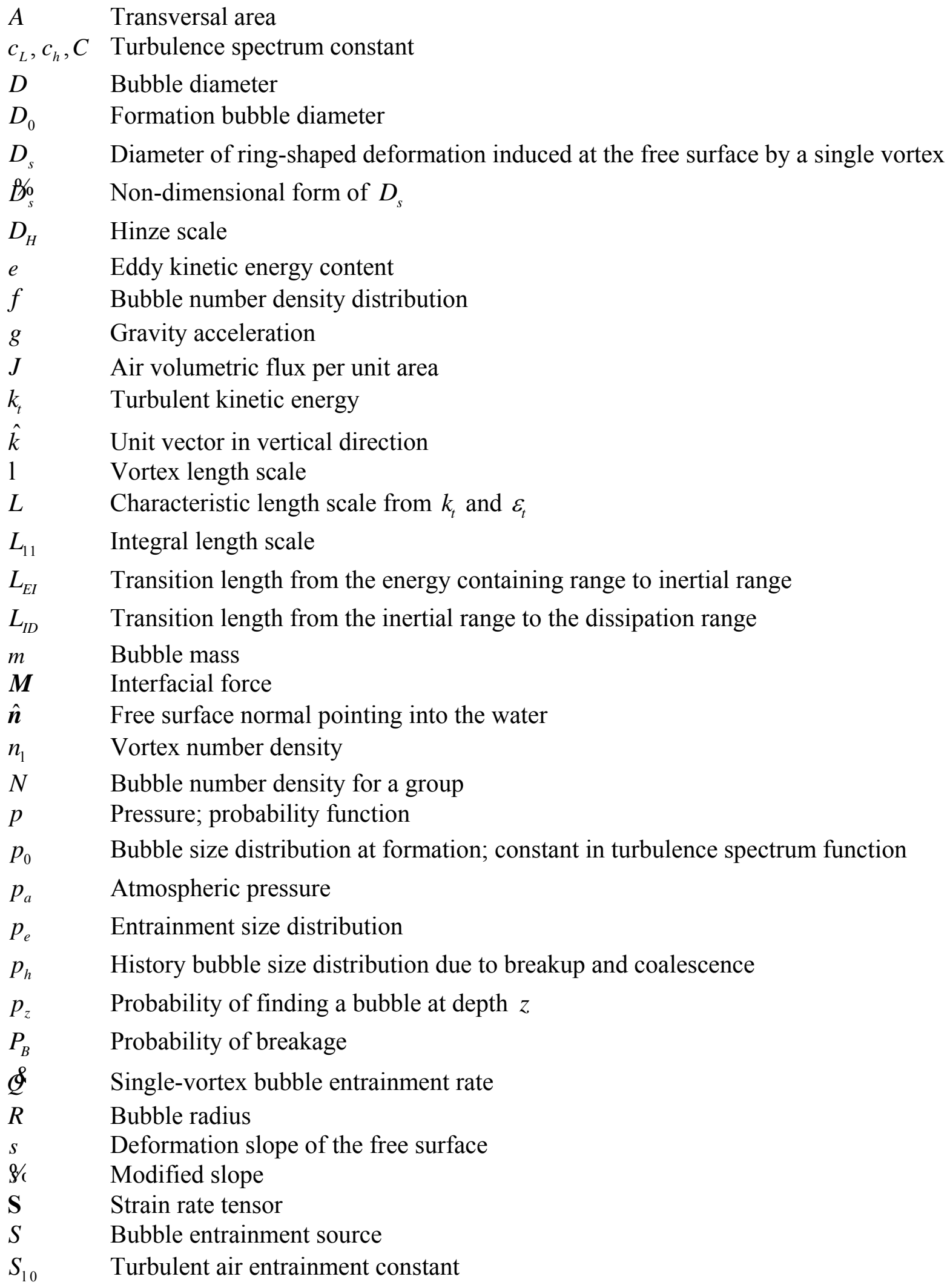




$\begin{array}{ll}t & \text { Time } \\ \mathrm{D} t & \text { Time step interval } \\ u & \text { Velocity vector } \\ u & \text { RMS of velocity fluctuation in turbulence } \\ \bar{v}_{0} & \text { Mean bubble volume at formation } \\ V_{T} & \text { Bubble terminal velocity } \\ d V, d V \phi & \text { Differential volume } \\ \mathrm{V} & \text { Single vortex configuration } \\ x & \text { Coordinate in } x \text { direction } \\ \& c & \text { Non-dimensional } x \text { coordinate } \\ y & \text { Coordinate in } y \text { direction } \\ y^{+} & \text {Viscous dimensionless wall distance } \\ z, z^{\prime} & \text { Depth under free surface } \\ z_{m} & \text { Minimum depth for a vortex to induce a } 45 \text { degree slope of on the free surface } \\ z_{e} & \text { For a single vortex, onset depth for entrainment } \\ d z, d z^{\prime} & \text { Differential depth }\end{array}$

\section{Dimensionless qroups}

\begin{tabular}{ll}
\hline$F r$ & Froude number \\
$W e$ & Weber number \\
$F r_{\ell}$ & Vortex Froude number \\
$R e_{l}$ & Taylor microscale Reynolds number \\
$S C$ & Bubble Schmidt number
\end{tabular}

\section{Greek letters}

\begin{tabular}{ll}
\hline$\alpha$ & Volume fraction \\
$\beta$ & Bubble breakup source; turbulence modeling constant \\
$d$ & Surface roughness \\
$\varepsilon_{t}$ & Turbulence dissipation \\
$\eta$ & Kolmogorov length scale \\
$\kappa$ & Wave number \\
$\lambda$ & Mixing length scale \\
$\mu$ & Dynamic viscosity \\
$\mu_{e f f}$ & Effective dynamic viscosity \\
$v$ & Kinematic viscosity \\
$v_{t 0}$ & Turbulent viscosity at the free surface \\
$\phi$ & Signed distance to the free surface (level set function) \\
$r$ & Mass density \\
$\sigma$ & Surface tension \\
$\chi$ & Bubble coalescence source
\end{tabular}




\section{Subscripts}

$d \quad$ Disperse phase

c Continuous phase

cr Critical value

$g \quad$ Group number

$i, j \quad$ Indices from 1 to 3

$m \quad$ Bubbles with mass $m$

min Minimum

M Maximum

$s \quad$ Free surface

$l \quad$ Mixing length model with length scale $l$

$\ell \quad$ Vortex with size $\ell$ 
Appendix: Implementation Summary.

This appendix summarizes the final set of equations needed to implement the proposed air entrainment model within a CFD code. Distance to the free surface is denoted by $\phi$, which in a level set method is readily available as part of the solution method. However, the model is not bound to any particular free surface representation as long as distance to the free surface is provided.

The final expression for the volumetric source of bubbles by air entrainment $S(D, z)$ is provided in Eq. (42). Once the entrainment size distribution $p_{e}(D)$ is specified (for instance from experiments), the only adjustable constant of the model is $S_{\ell 0}$. The individual terms required to evaluate $S(D, z)$ in Eq. (42) are:

1. The history size distribution $p_{h}(D)$, estimated from the entrainment size distribution $p_{e}(D)$ Eq. (44).

2. The phase fraction $\Lambda\left(\alpha_{d}\right)=1-\alpha_{d}$, defined after Eq. (16) in $\S 2.2 .3$.

3. The probability of finding a bubble at a given depth $P_{z}(z \mid D)$, Eq. (37). For implementation within a CFD code, the depth $z$ is replaced by the distance to the free surface $\phi$. This distance is readily available when using level-set approaches as described in $\$ 2.1$.

4. The mean bubble volume $\bar{v}_{0}$, is obtained from the experimental size distribution as described at the end of $\S 2.3 .4$.

5. The total flux of entrained air $J$, Eq. (42):

a. The integral in Eq. (42) is computed numerically. Practical limits of integration were found in the study of Fig. 9. The lower limit is taken to be a fraction Hinze's diameter as $\ell_{\min }=D_{H} / 10$. The upper limit is taken to be proportional to the integral length scale (from $\S 2.2 .2 L_{11} \approx 0.43 k_{t}^{3 / 2} / \varepsilon_{t}$ ) as $\ell_{\max }=10 L_{11}$.

b. The one dimensional integral for $J$ is best performed in a logarithmic scale as described by Eq. (43).

c. The terms in the integrand of Eq. (42) for $J$ are:

i. $\quad$ The probability of breakage $P_{B}$ from Eq. (30) with $u_{\ell}$ from Eq. (14).

ii. Integral $I_{Q}$ for the total rate of entrainment for a single vortex, Eq. (41).

iii. Turbulence spectrum modulation $f_{L}$, Eq. (12).

iv. Onset depth of entrainment $z_{e}$ for a single vortex, Eq. (25). 
Table 1: Turbulence characteristics at Athena's stern.

\begin{tabular}{|l|l|c|}
\hline Scale & Symbol [unit] & Value \\
\hline Turbulent intensity & $I_{t}[-]$ & 0.2 \\
\hline Integral length scale & $L_{11}[\mathrm{~m}]$ & 0.3 \\
\hline RMS velocity & $u_{0}[\mathrm{~m} / \mathrm{s}]$ & 1.08 \\
\hline Turbulent kinetic energy & $k_{t}\left[\mathrm{~m}^{2} / \mathrm{s}^{2}\right]$ & 1.75 \\
\hline Turbulent dissipation & $\varepsilon_{t}\left[\mathrm{~m}^{2} / \mathrm{s}^{3}\right][\mathrm{W} / \mathrm{Kg}]$ & 3.32 \\
\hline Turbulent viscosity & $v_{t}\left[\mathrm{~m}^{2} / \mathrm{s}\right]$ & 0.0831 \\
\hline Kolmogorov scale & $\eta[\mathrm{m}]$ & $23.43 \times 10^{-6}$ \\
\hline Hinze's diameter & $D_{H}[\mathrm{~m}]$ & $1.47 \times 10^{-3}$ \\
\hline
\end{tabular}


Table 2: Representative cell sizes for the grid study for Athena R/V.

\begin{tabular}{|l|c|c|c|c|}
\hline \multirow{2}{*}{ Grid } & \multirow{2}{*}{$\begin{array}{c}\text { Million } \\
\text { Grid Points }\end{array}$} & \multicolumn{3}{|c|}{ Grid Spacing [mm] } \\
\cline { 3 - 5 } & 17.0 & Bow $(d x)$ & Stern & BL $\left(y^{+}\right)$ \\
\hline Very Coarse & $34.0(27.0)$ & 50.0 & $0.22(34)$ \\
\hline Coarse & 34.0 & $3.2(21.4)$ & 39.7 & $0.17(27)$ \\
\hline Medium & 67.6 & $2.5(17.0)$ & 31.5 & $0.14(21)$ \\
\hline Fine & 135.8 & $2.0(13.5)$ & 25.0 & $0.11(17)$ \\
\hline Very Fine & 268.8 & $1.6(10.7)$ & 19.9 & $0.08(14)$ \\
\hline
\end{tabular}


Table 3: Results for the total turbulent bubble entrainment source at the stern of Athena $\mathrm{R} / \mathrm{V}$ for five grids.

\begin{tabular}{|l|c|c|c|}
\hline Grid & $\mathrm{D} x / \mathrm{D} x_{0}$ & $S_{\text {int }}[-]$ & $\begin{array}{c}\text { Relative Error } e_{\mathrm{D}} \\
{[\%]}\end{array}$ \\
\hline VCoarse & 1 & $1.7173 \mathrm{E}-05$ & \\
\hline Coarse & 0.793701 & $1.7238 \mathrm{E}-05$ & $3.81 \mathrm{E}-01$ \\
\hline Medium & 0.629961 & $1.6828 \mathrm{E}-05$ & $2.38 \mathrm{E}+00$ \\
\hline Fine & 0.5 & $1.6682 \mathrm{E}-05$ & $8.71 \mathrm{E}-01$ \\
\hline VFine & 0.39685 & $1.6619 \mathrm{E}-05$ & $3.76 \mathrm{E}-01$ \\
\hline
\end{tabular}

Table 4: Grid study summary for the total turbulent bubble entrainment source at the stern of Athena $\mathrm{R} / \mathrm{V}$.

\begin{tabular}{|c|c|c|c|c|c|}
\hline Grid System & $\boldsymbol{P}_{\boldsymbol{G}}$ & $\boldsymbol{C}_{\boldsymbol{G}}$ & $\begin{array}{c}\boldsymbol{\delta}_{\boldsymbol{G}}^{*} \\
(\boldsymbol{\%} \mathbf{V})\end{array}$ & $\begin{array}{c}\boldsymbol{U}_{\boldsymbol{G}} \\
(\mathbf{\% V F})\end{array}$ & $\begin{array}{c}\text { Convergence } \\
\text { Type }\end{array}$ \\
\hline VCoarse/Medium/VFine & 1.08 & 0.43 & 1.93 & 1.71 & Monotonic \\
\hline Coarse/Medium/Fine & 2.23 & 1.19 & 0.67 & 0.09 & Monotonic \\
\hline Medium/Fine/VFine & 1.82 & 0.87 & 0.29 & 0.04 & Monotonic \\
\hline
\end{tabular}




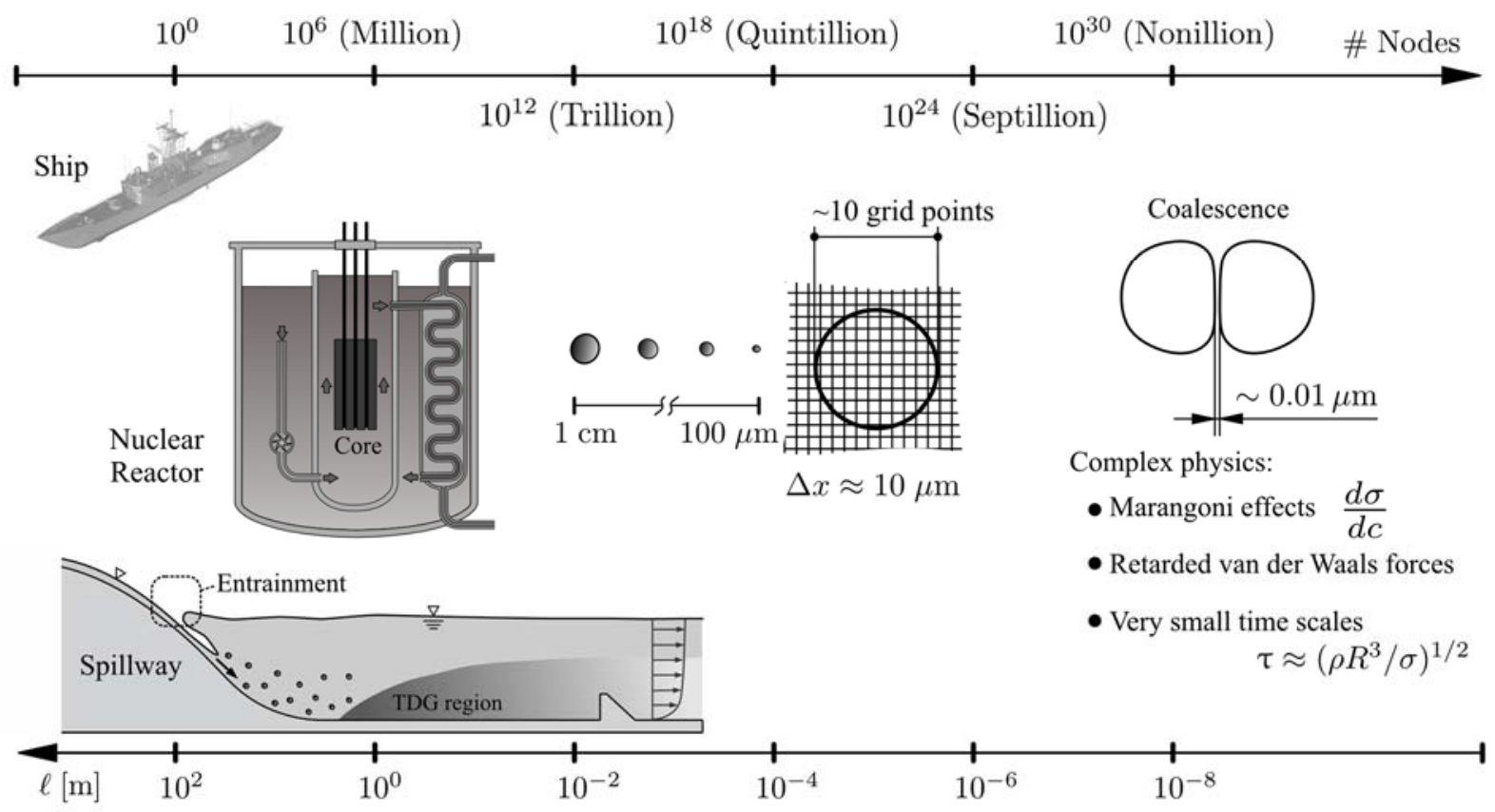

Figure 1: Wide range of scales in bubbly flows with air entrainment from the film breakup process during coalescence to the full scale of the problem of interest. 


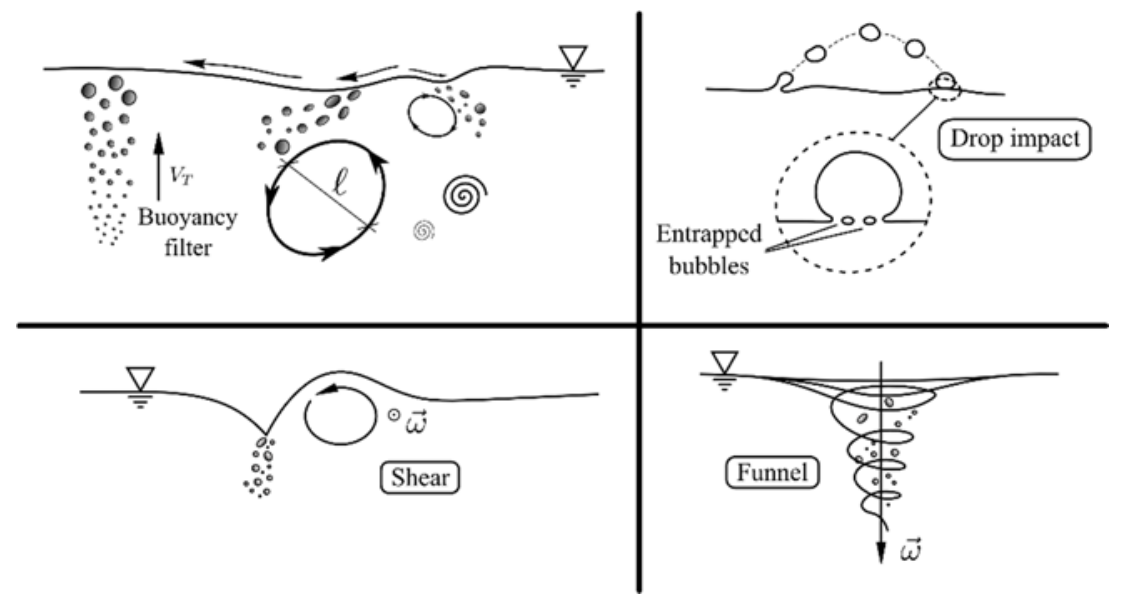

Figure 2: Some of the physical processes that lead to bubble entrainment. 


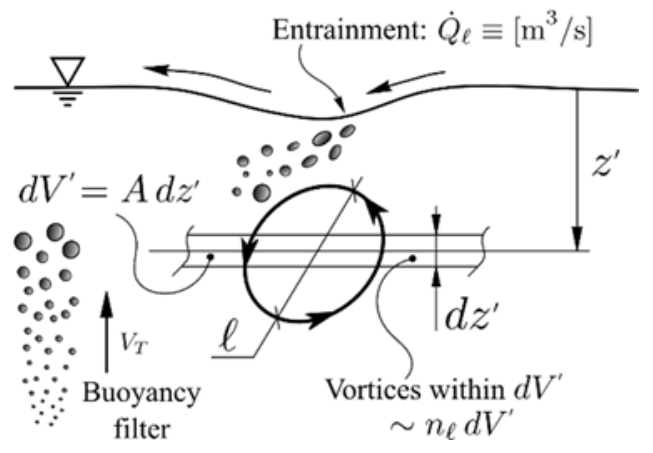

Figure 3: Model idealization of the single vortex contribution to air entrainment. 


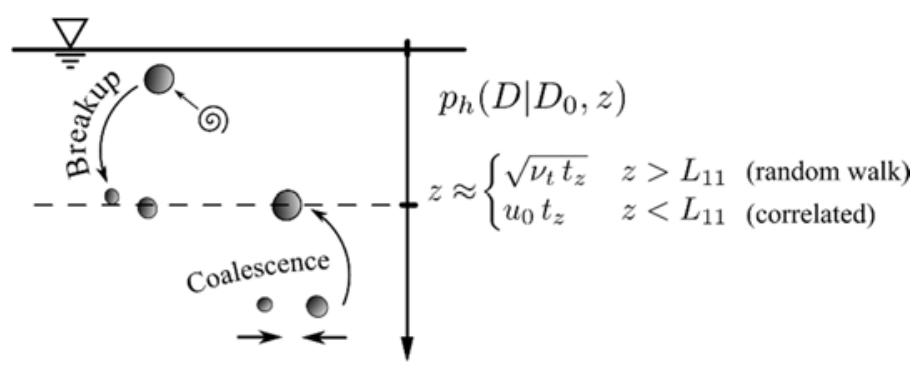

Figure 4: Bubble history as it travels from its inception at the free surface to depth $z$ in time $t_{z}$. 


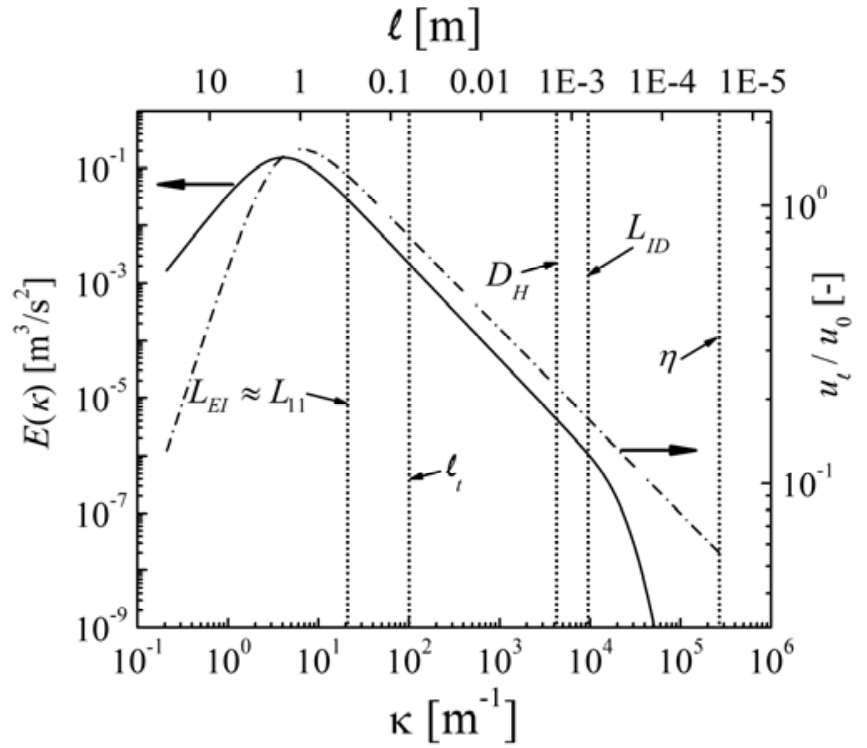

Figure 5: CFD estimation of turbulent scales at the stern of a $47 \mathrm{~m}$ ship. 


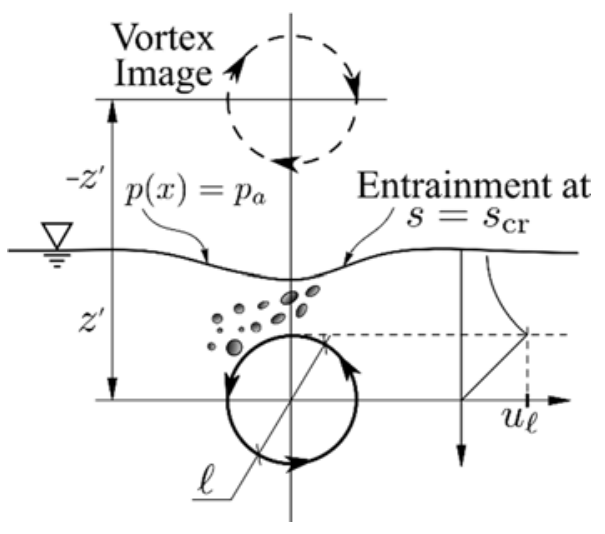

Figure 6: Simplified potential flow model for single vortex contribution to air entrainment. 

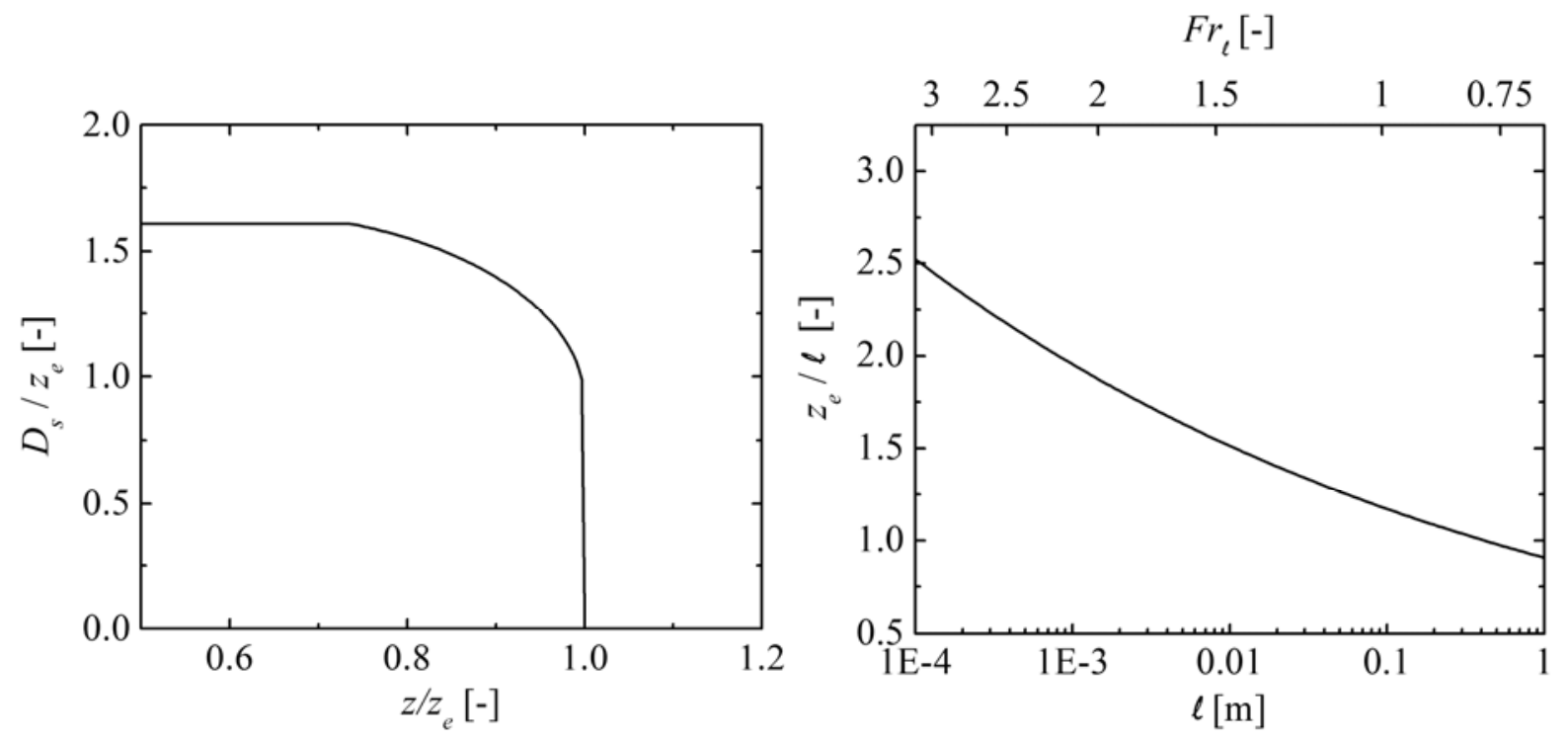

Figure 7: Induced entrainment ring for a vortex of size $\ell$. a) Ring diameter. b) Onset of entrainment depth. 


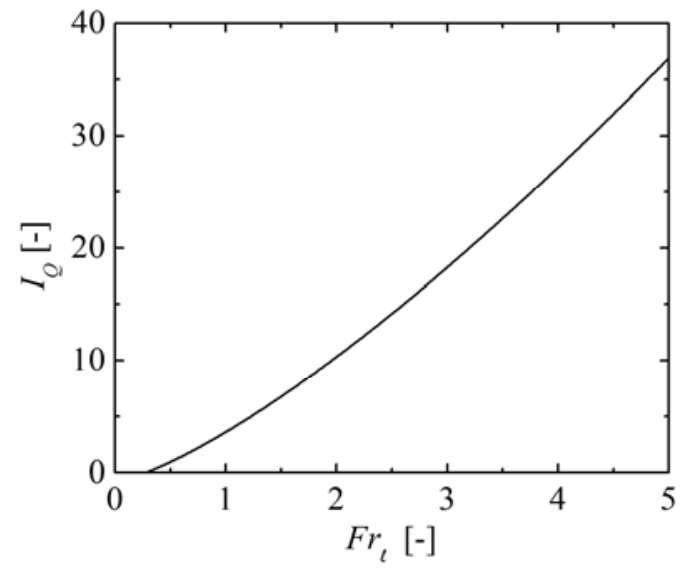

Figure 8: Single vortex entrainment source integrated in the depth coordinate. 


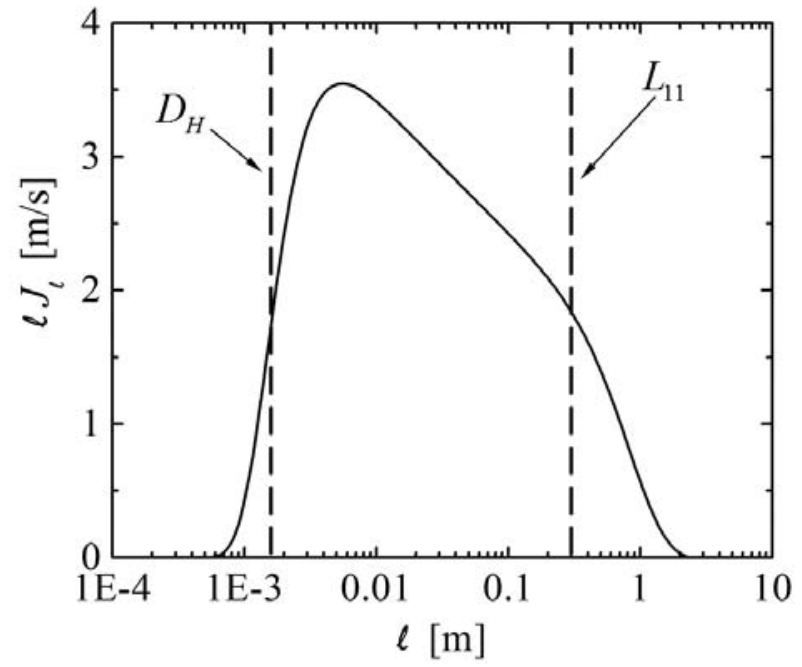

Figure 9: Spectral contribution to the total entrainment flux as a function of vortex size. 

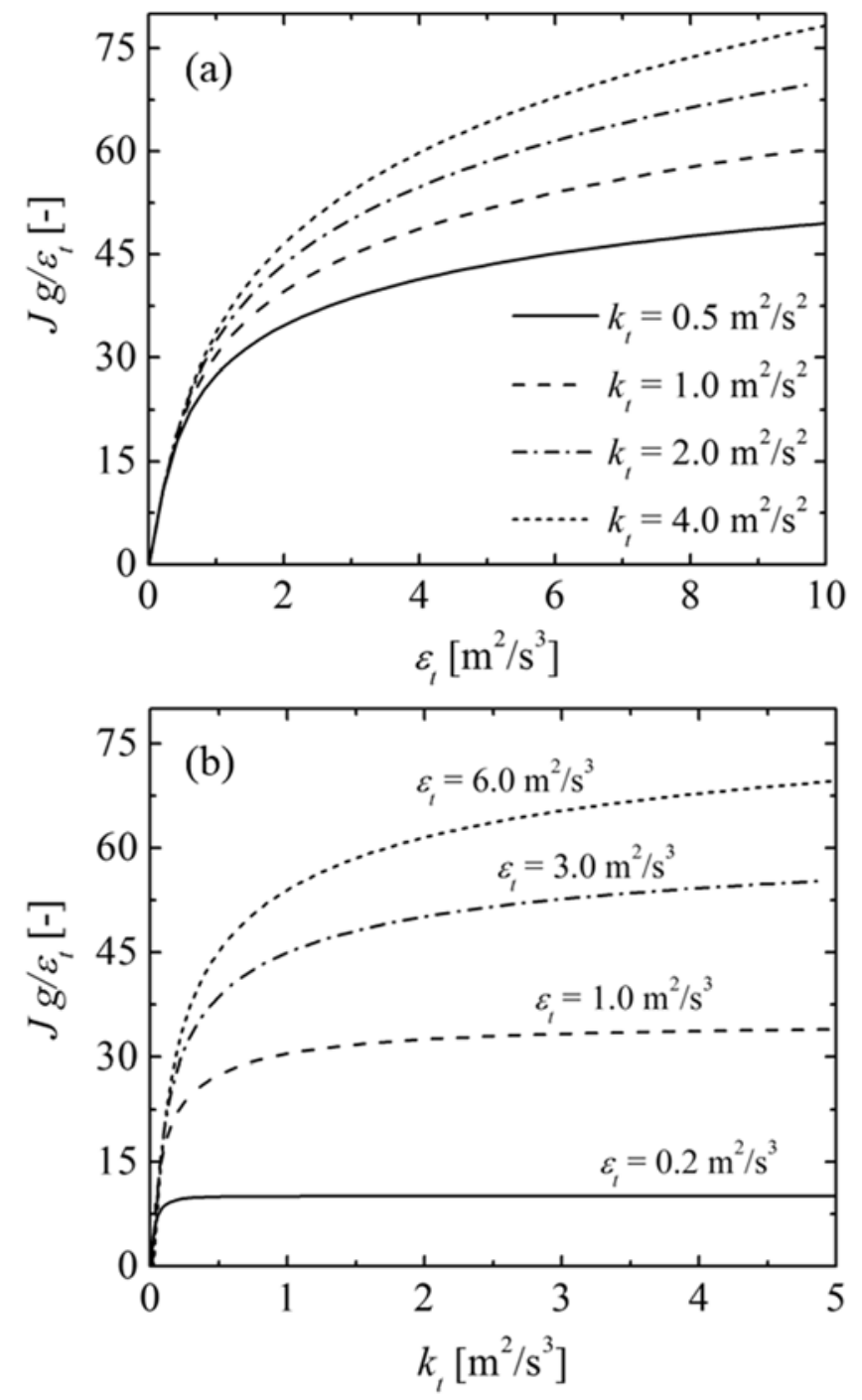

Figure 10: Total air entrainment flux as a function of turbulent dissipation (a) and turbulent kinetic energy (b). 


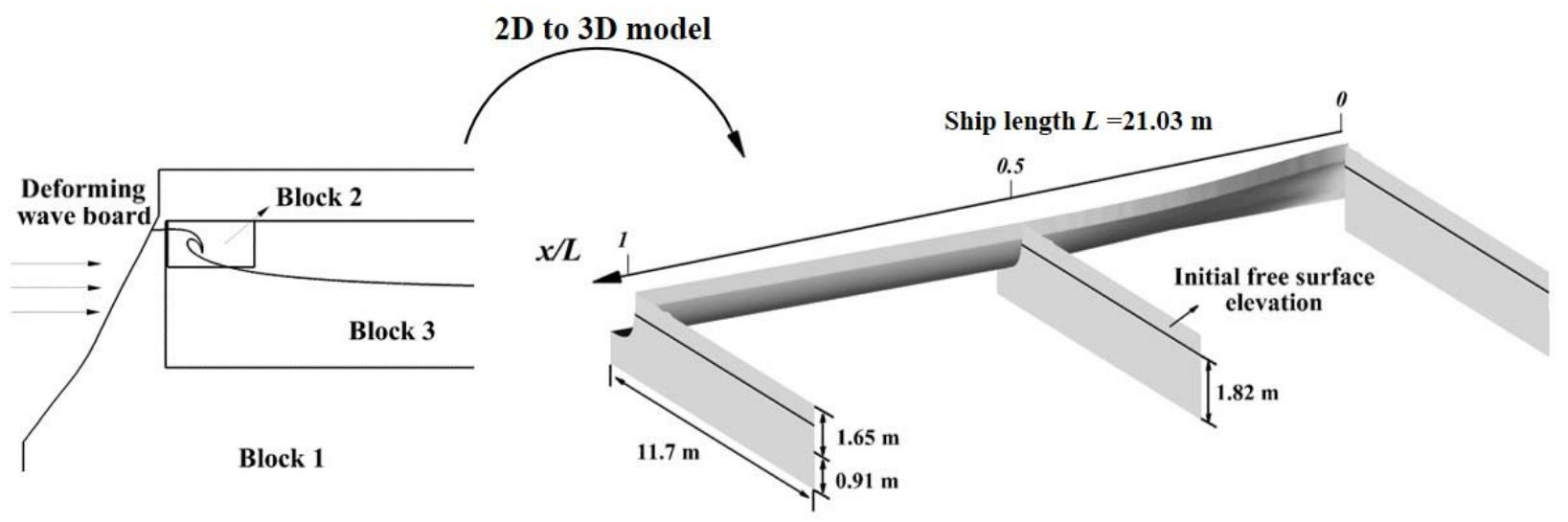

Figure 11: Overset grid system for the $2 \mathrm{D}+\mathrm{T}$ wave maker simulation. 


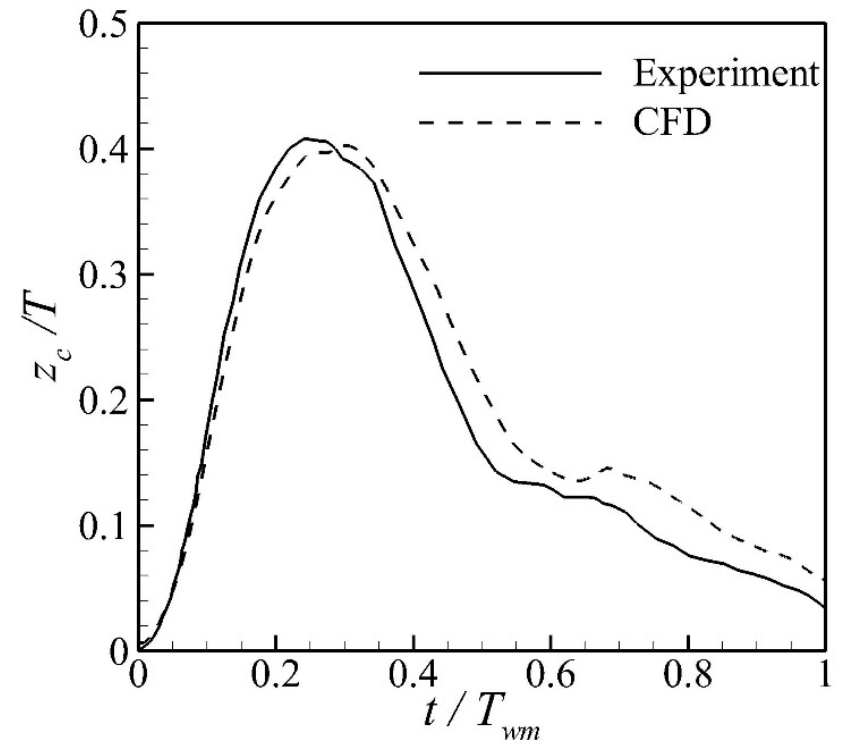

Figure 12: Time evolution of the surface elevation on the wave board. 

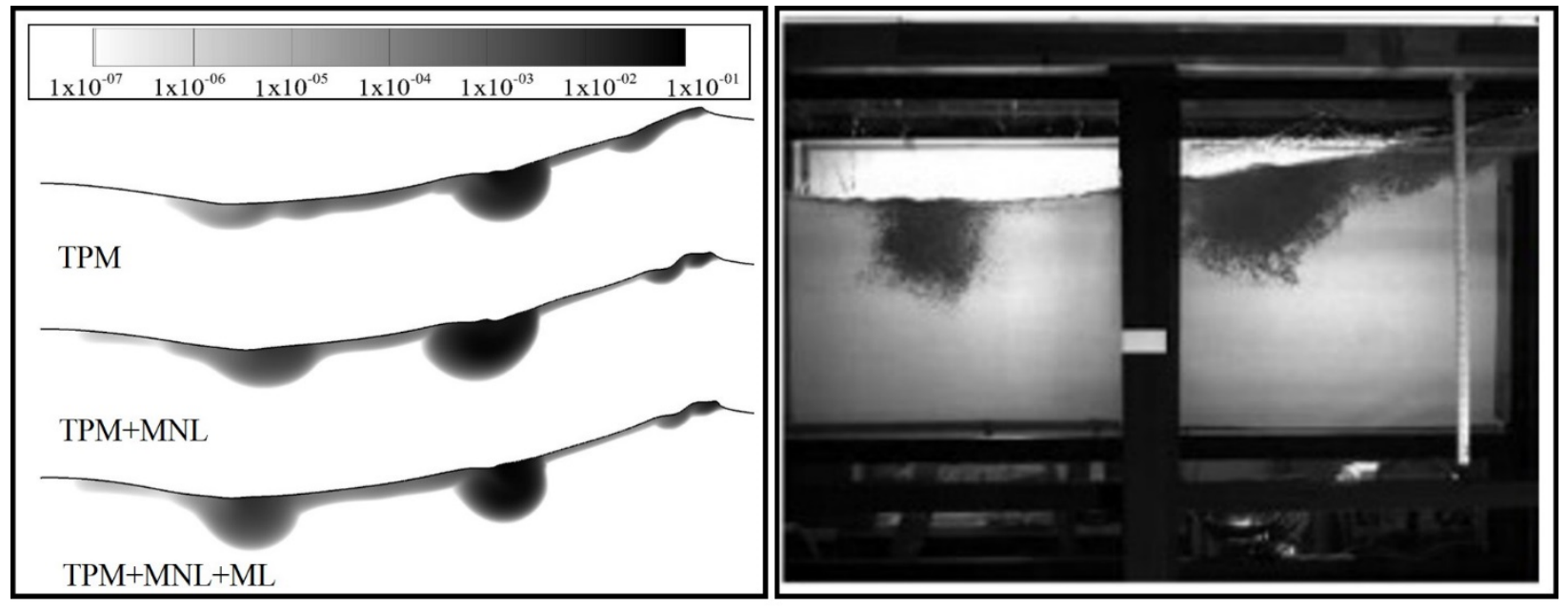

Figure 13: Void fraction contours at $x / L=0.5$ using the three proposed turbulence model modifications. Experimental clouds are shown for comparison (Tavakolinejad, 2010). 


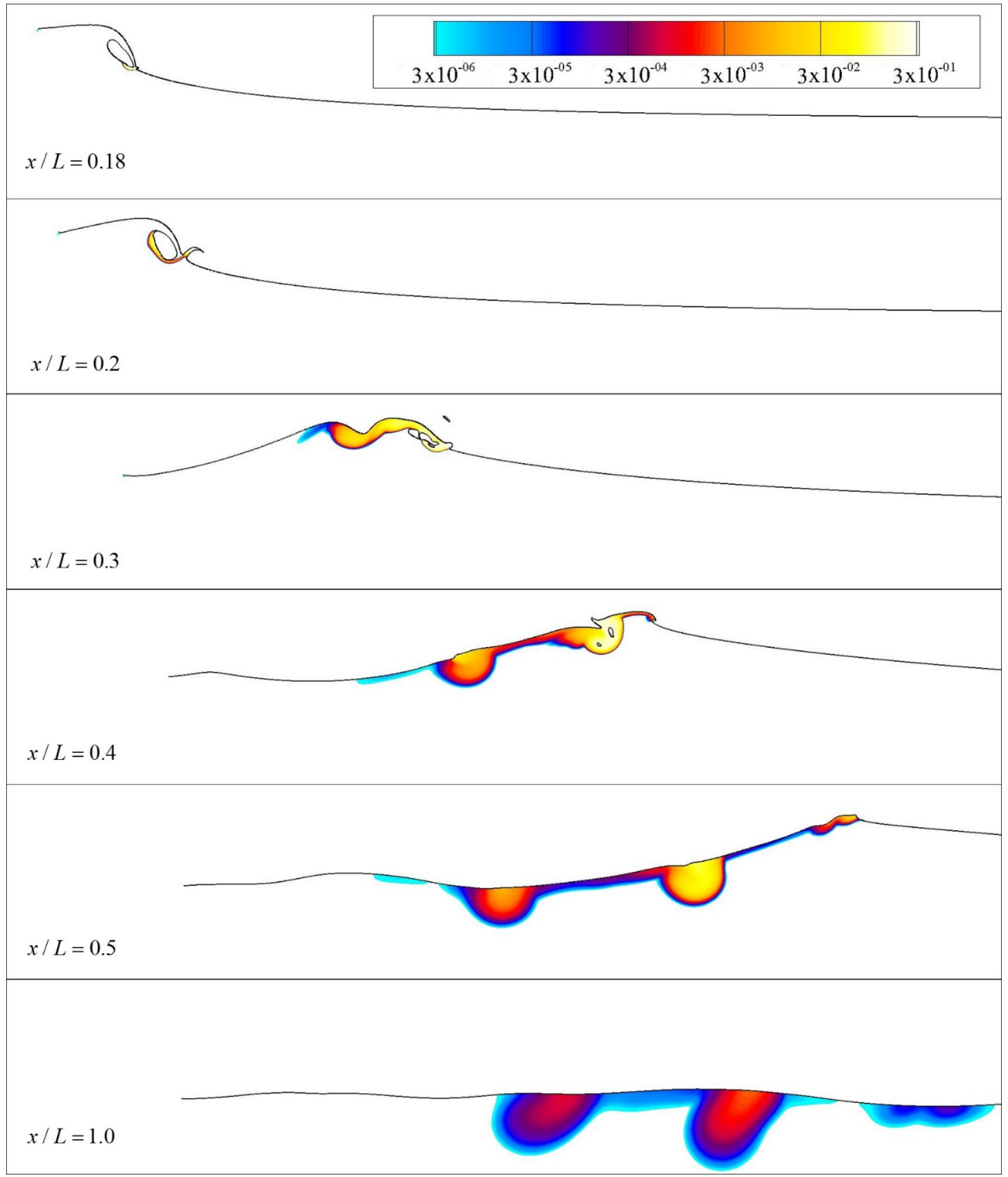

Figure 14: Void fraction as the breaking wave is formed and evolves into two bubble clouds. 


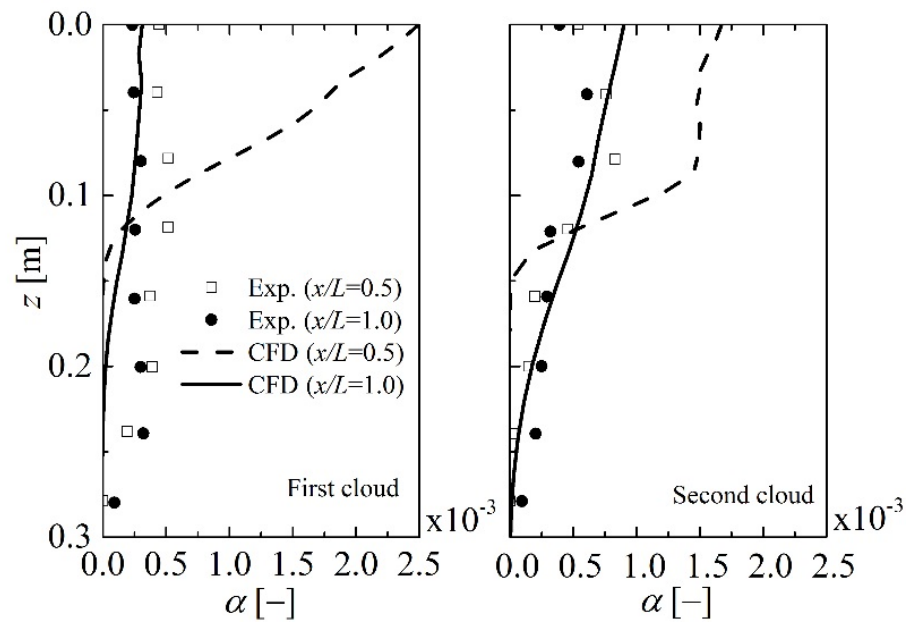

Figure 15: Void fraction profiles versus depth for the first and second clouds. The predicted void fraction on the second cloud at $x / L=0.5$ is divided by a factor of 20 to fit the axes in the figure. 

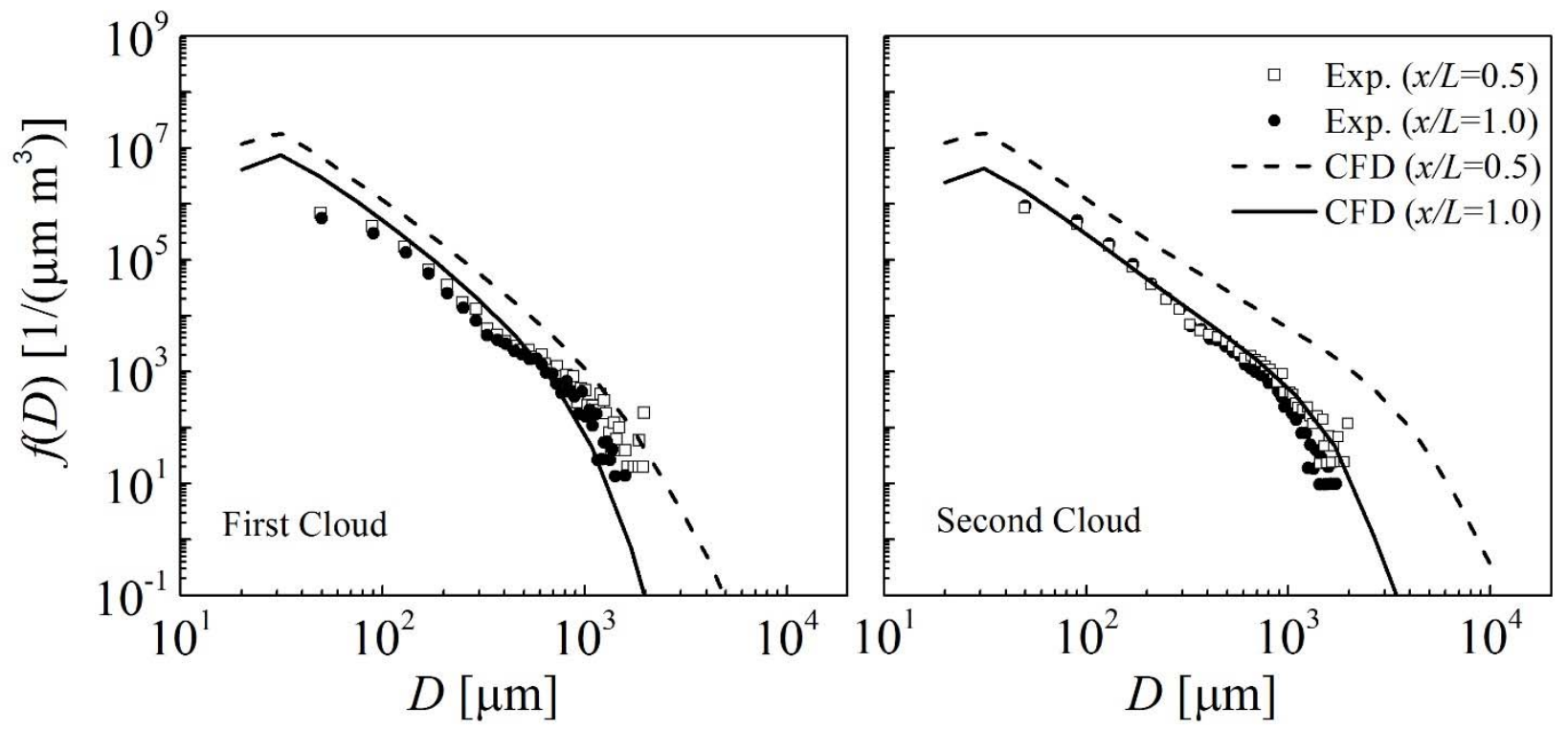

Figure 16: Number density distribution for first and second clouds. 

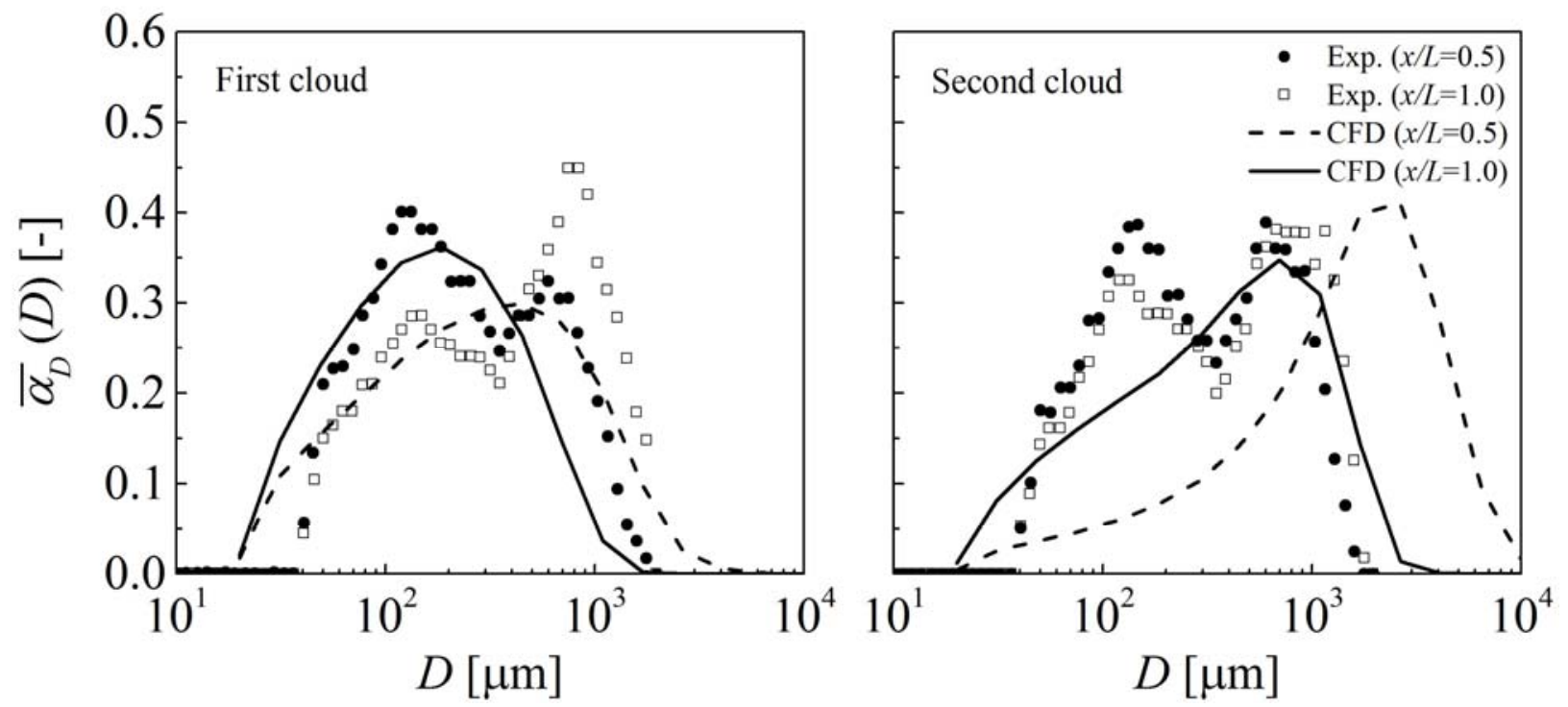

Figure 17: Normalized void fraction size distribution for first and second clouds. 


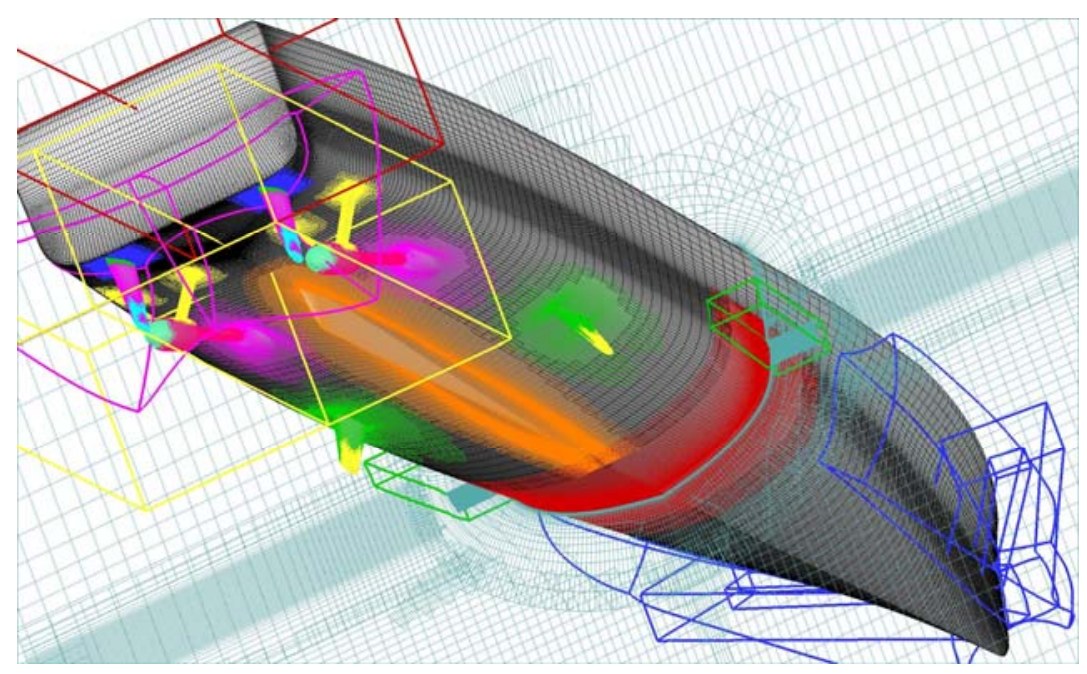

Figure 18: Overset grid system for the fully appended Athena R/V showing refinement blocks as colored boxes. At slice at midship (cyan) shows the overlap among the background, free surface refinement and boundary layer grids. 


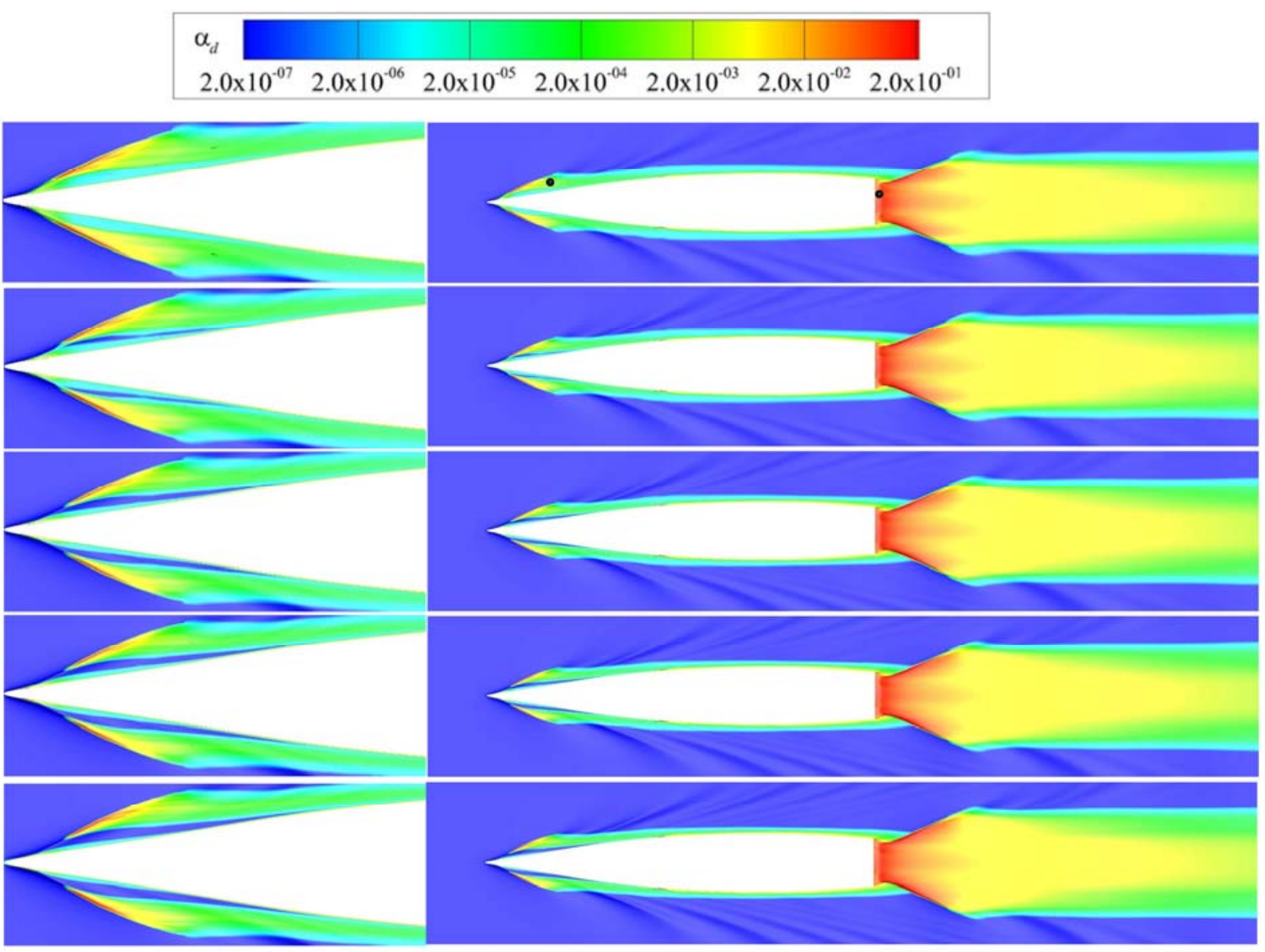

Figure 19: Free surface colored with void fraction for Athena R/V advancing at 10.5 knots. From top to bottom: VCoarse, Coarse, Medium, Fine, and VFine grids. 


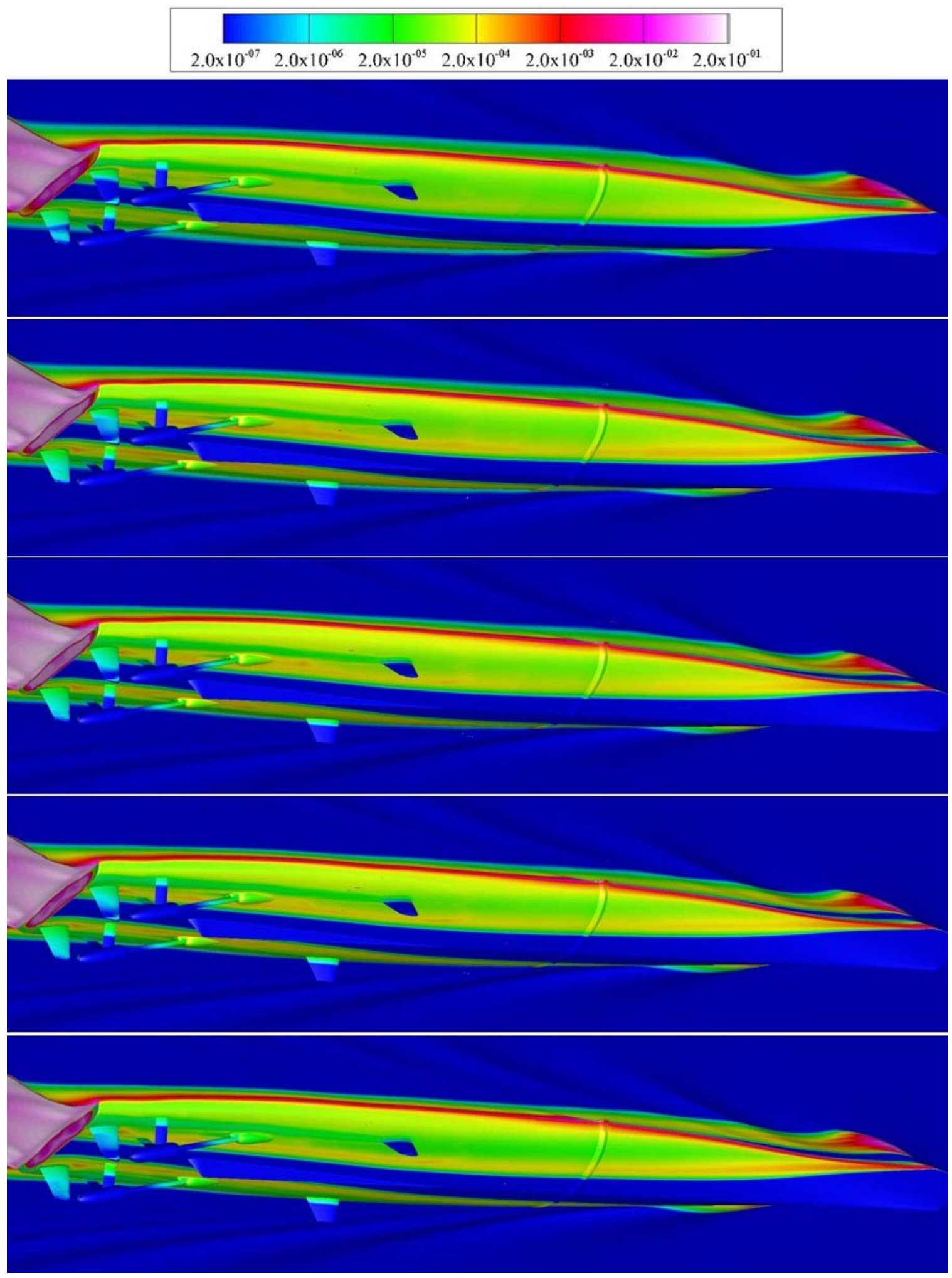

Figure 20: Void fraction contours on the hull of Athena $\mathrm{R} / \mathrm{V}$ and free surface. From top to bottom: VCoarse, Coarse, Medium, Fine, and VFine grids. 


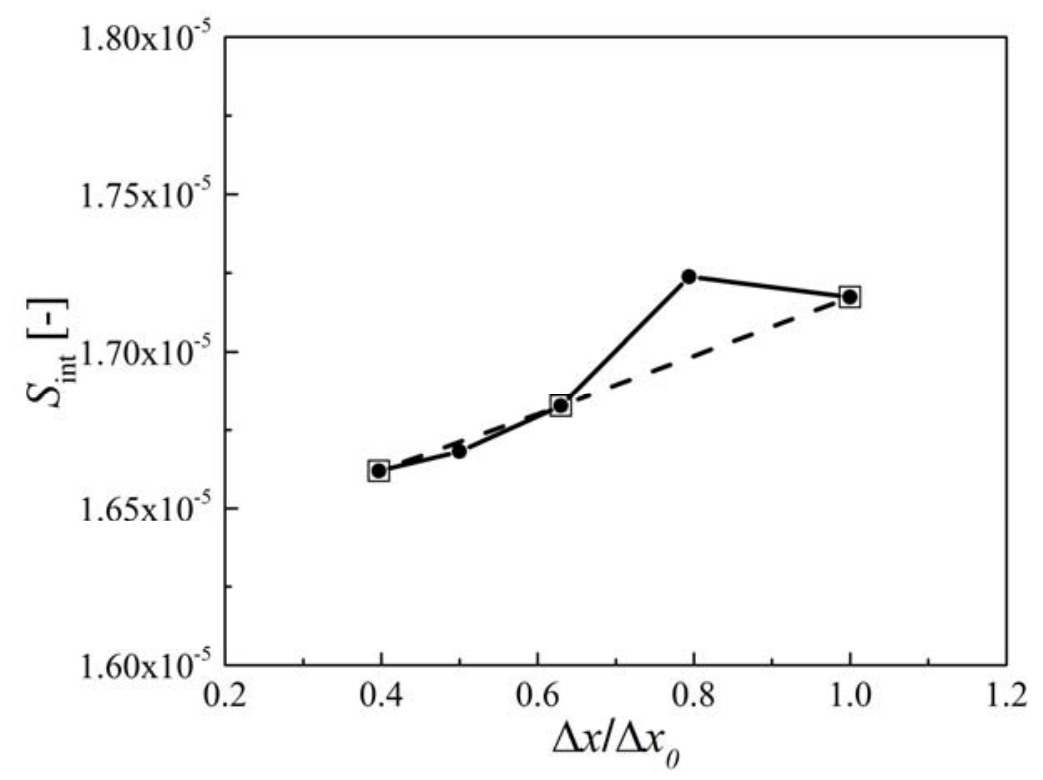

Figure 21: Total entrainment source at the stern of Athena $R / V$ as a function of grid spacing. 


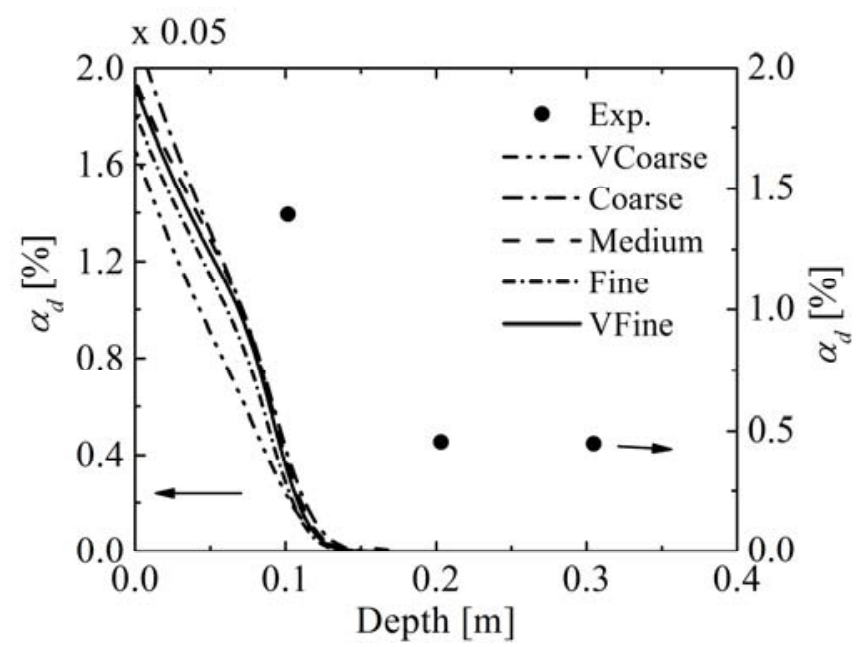

Figure 22: Void fraction as a function of depth at the bow. 


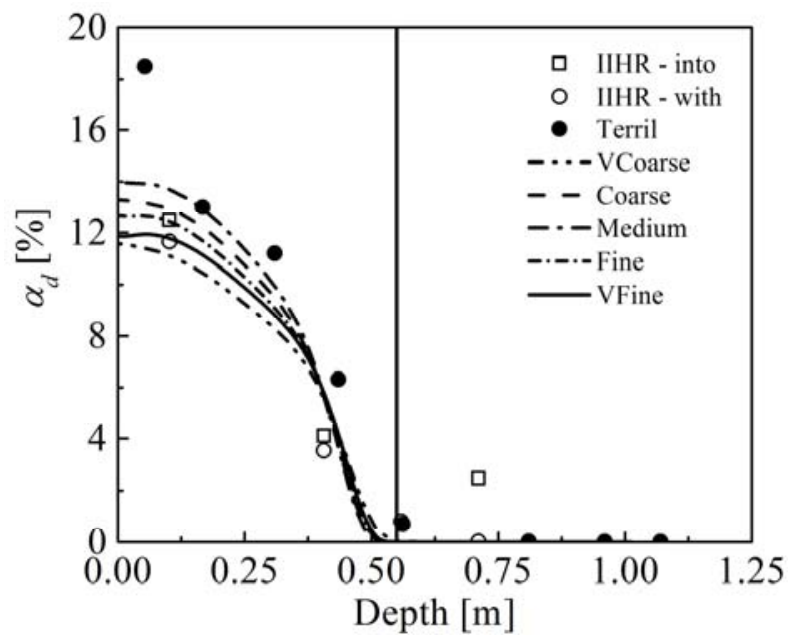

Figure 23: Void fraction as a function of depth at the stern. The black vertical line indicates the bottom line of the transom. 

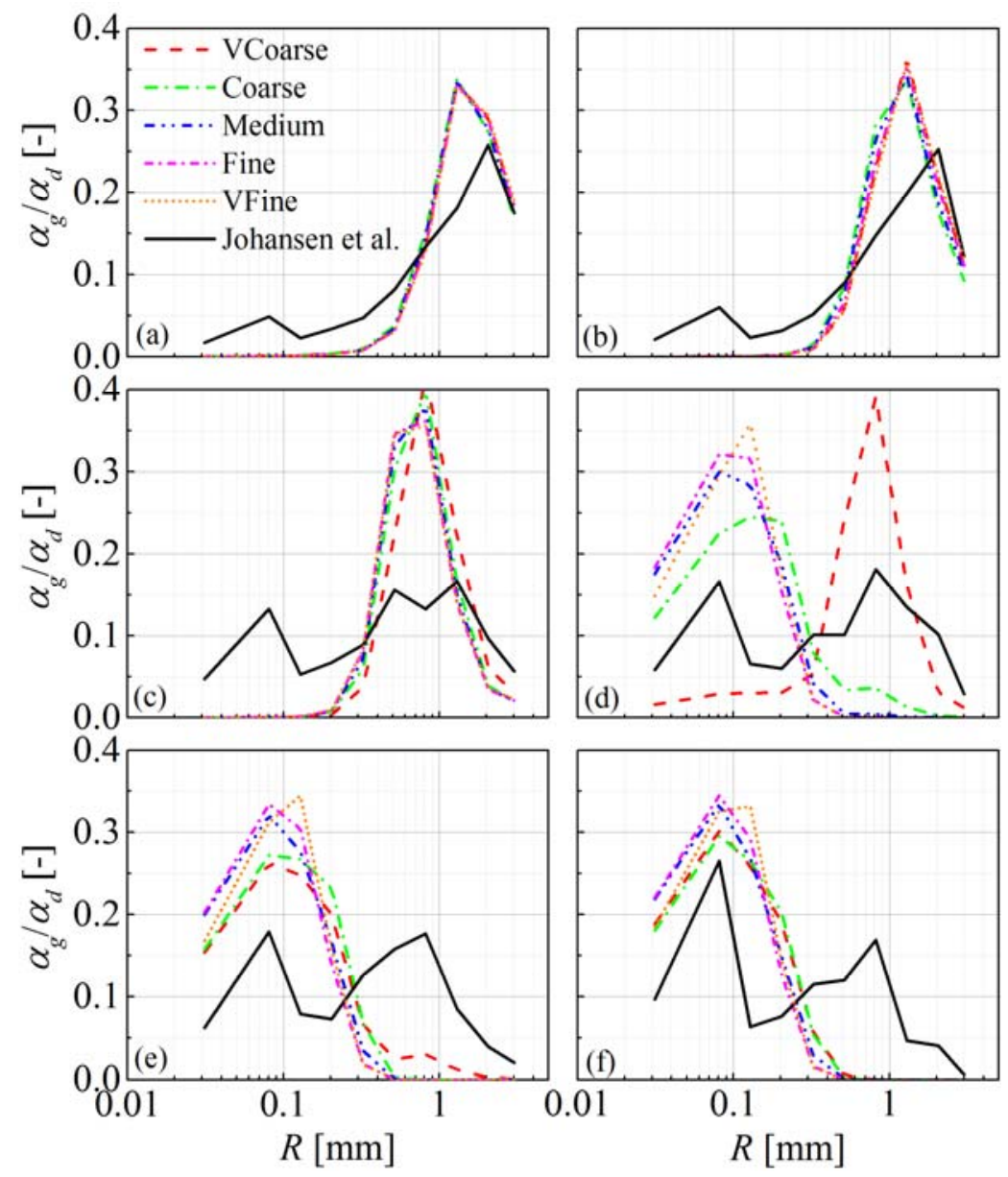

Figure 24: Normalized void fraction for experimental groups at different depths. (a) to (f) correspond to $0.1 \mathrm{~m}, 0.3 \mathrm{~m}, 0.5 \mathrm{~m}, 0.6 \mathrm{~m}, 0.65 \mathrm{~m}, 0.7 \mathrm{~m}$ in depth. 